\title{
Supersymmetry and the Parisi-Sourlas Dimensional Reduction: A Rigorous Proof
}

\author{
Abel Klein ${ }^{1, \star}$, Lawrence J. Landau ${ }^{2}$, and J. Fernando Perez ${ }^{3, \star \star}$ \\ 1 Department of Mathematics, University of California, Irvine, CA 92717, USA \\ 2 Mathematics Department, Bedford College, University of London, London NW1 4NS, \\ England \\ 3 Instituto de Física, Universidade de São Paulo, Caixa Postal 20516, São Paulo, S.P., Brazil
}

\begin{abstract}
Functional integrals that are formally related to the average correlation functions of a classical field theory in the presence of random external sources are given a rigorous meaning. Their dimensional reduction to the Schwinger functions of the corresponding quantum field theory in two fewer dimensions is proven. This is done by reexpressing those functional integrals as expectations of a supersymmetric field theory. The Parisi-Sourlas dimensional reduction of a supersymmetric field theory to a usual quantum field theory in two fewer dimensions is proven.
\end{abstract}

It was observed in the physics literature [1-5] that a $D$-dimensional classical field theory coupled to an external random source having Gaussian correlations is related order by order in perturbation theory to the corresponding $(D-2)$ dimensional quantum field theory without external sources.

This connection between random systems and the corresponding pure systems in two fewer dimensions was first noticed by Imry and Ma [1]. Investigating the effect of a quenched random magnetic field on phase transitions, they argued that classical mean field behavior occurred for dimensions greater than 6 , instead of 4 as in non-random systems. Grinstein [2] found that the scaling laws for these random systems are the same as for pure systems, except that the dimension $D$ is replaced by $D+2$.

Aharony, Imry, and Ma [3] explained those findings by arguing that the Feynman diagrams which give the leading singular behavior for the random case are identically equal, apart from combinatorial factors, to the corresponding Feynman diagrams for the pure case in two fewer dimensions. They showed this for one-loop diagrams that remain connected after all lines with random source insertions are opened. Young [4] extended their result for similar diagrams with an arbitrary number of loops.

* Partially supported by the NSF under grant MCS-8301889

$\star \star$ Partially supported by FAPESP 
The situation was clarified by Parisi and Sourlas [5]. They observed that the most infrared divergent diagrams are those with the maximum number of random source insertions, and, if the other diagrams are neglected, one is left with a diagrammatic expansion for a classical field theory in the presence of random sources.

Parisi and Sourlas then pointed out that the underlying phenomenon for the connection between random systems and pure systems in two fewer dimensions is that a classical field theory in the presence of random sources is perturbatively equivalent to the corresponding quantum field theory in two fewer dimensions. Parisi and Sourlas explained this dimensional reduction by a hidden supersymmetry.

Niemi [6] has rewritten the Parisi and Sourlas argument for the dimensional reduction in terms of the formal generating function, McClain, Niemi, Taylor, and Wijewardhana [7] extended Parisi and Sourlas' results to non-scalar theories, including gauge and fermionic field theories.

Non-perturbative versions of the Parisi and Sourlas argument have been given by Klein and Perez [8] and by Cardy [9].

In this article we give a rigorous proof of the Parisi-Sourlas dimensional reduction.

We start by discussing the average correlation functions of a classical field theory in the presence of random external sources which are distributed as white noise (i.e., they have "Gaussian correlations"). Parisi and Sourlas rewrote those average correlation functions as functional integrals by means of a formal change of variables. (It should be noticed that even at the formal level this change of variables can only be justified when we have uniqueness for the solution of the classical field equation and that the connection of these functional integrals with the average correlation functions remain unclear in the non-uniqueness case; this has been discussed by Parisi and Sourlas [10].) We construct rigorously those functional integrals and take them as the definition of the average correlation functions. To do that we introduce the appropriate cutoffs. It turns out that the space cutoff must not only satisfy certain decay properties so the formal positivity of certain terms is preserved, but a counterterm depending on the boundary of the space cutoff must be added to preserve the hidden supersymmetry that is responsible for the dimensional reduction.

Next we construct supersymmetric field theories and show the dimensional reduction of a supersymmetric field theory to a usual quantum field theory in two fewer dimensions. To avoid technical problems in the integration over anticommuting variables a momentum cutoff is introduced. This cutoff turns out to be determined by the requirements that the supersymmetry be preserved and that a certain formal positivity be also preserved.

Finally, it is shown that expectations of this supersymmetric field theory with the momentum cutoff give an approximation to the average correlation functions we started with. Removing the cutoff we obtain the dimensional reduction for the average correlation functions.

This article is organized as follows:

1. The Problem. The dimensional reduction is stated at the formal level

2. Rigorous Results. Contains the statements of Theorems I and II. Theorem I is the construction of the average correlation functions. Theorem II is the dimensional reduction of the average correlation functions 
3. Proof of Theorem I

4. Supersymmetries: Some Basic Facts. Lemma 4.5 is the key to the dimensional reduction

5. Supersymmetric Free Field Theories

6. Supersymmetric Interactions

7. The Dimensional Reduction for Supersymmetric Theories. The precise statement is Theorem III

8. Proof of Theorem II

Before we start, let us fix some notation that will be used throughout this article: $z, x, y$ will always denote elements of $\mathbb{R}^{D}, \mathbb{R}^{D-2}, \mathbb{R}^{2}$, respectively. We will also write $z=(x, y)$.

Similarly, in momentum space $q, p, k$ will always denote elements of $\mathbb{R}^{D}, \mathbb{R}^{D-2}, \mathbb{R}^{2}$, respectively, and $q=(p, k)$.

We will also use the notation $z^{2}=z \cdot z, y^{2}=y \cdot y$, etc.

\section{The Problem}

Let us consider a classical scalar field theory in $D$ dimensions with Lagrangian

$$
\mathscr{L}(\varphi)=\frac{1}{2}(\nabla \varphi)^{2}+\frac{1}{2} m^{2} \varphi^{2}+V(\varphi),
$$

and let $\mathscr{L}_{h}(\varphi)=\mathscr{L}(\varphi)+h \varphi$ be the Lagrangian in the presence of an external source $h$.

The classical equation of motion is

$$
\left(-\Delta+m^{2}\right) \varphi+V^{\prime}(\varphi)=-h .
$$

The external sources are taken to be random with "Gaussian correlations", i.e., $\left\{h(z) ; z \in \mathbb{R}^{D}\right\}$ form a generalized Gaussian system with mean zero and covariance

$$
\left\langle h(z) h\left(z^{\prime}\right)\right\rangle=\gamma \delta\left(z-z^{\prime}\right),
$$

where $\gamma>0$ is a given constant.

Proceeding formally, let $\varphi_{h}$ denote the formal solution to (1.2) given by perturbation theory and define the average correlation functions

$$
R\left(z_{1}, \ldots, z_{n}\right)=\left\langle\varphi_{h}\left(z_{1}\right) \ldots \varphi_{h}\left(z_{n}\right)\right\rangle,
$$

the average being taken over the random sources $h$, i.e., formally

$$
R\left(z_{1}, \ldots, z_{n}\right)=\frac{\int \varphi_{h}\left(z_{1}\right) \ldots \varphi_{h}\left(z_{n}\right) \exp \left\{-(1 / 2 \gamma) \int h(z)^{2} d z\right\} \mathscr{D} h}{\int \exp \left(\left\{-(1 / 2 \gamma) \int h(z)^{2} d z\right\} \mathscr{D} h\right.} .
$$

Let us now consider a Euclidean quantum field theory in $D-2$ dimensions with Lagrangian $(4 \pi / \gamma) \mathscr{L}(\phi)$, where $\mathscr{L}$ is the same Lagrangian given by (1.1). Let

$$
S\left(x_{1}, \ldots, x_{n}\right)=\frac{\int \phi\left(x_{1}\right) \ldots \phi\left(x_{n}\right) \exp \left\{-(4 \pi / \gamma) \int \mathscr{L}(\phi(x)) d x\right\} \mathscr{D} \phi}{\int \exp \left\{-(4 \pi / \gamma) \int \mathscr{L}(\phi(x)) d x\right\} \mathscr{D} \phi}
$$

be the Schwinger functions of the theory.

Parisi and Sourlas [5] argued that

$$
R\left(\left(x_{1}, 0\right), \ldots,\left(x_{n}, 0\right)\right)=S\left(x_{1}, \ldots, x_{n}\right),
$$

order by order in perturbation theory. 
To explain (1.4), Parisi and Sourlas started by rewriting $R\left(z_{1}, \ldots, z_{n}\right)$ as an integral over the field $\varphi$. This is done by performing on the right-hand-side of (1.3) the change of variables given by (1.2); formally, we get

$$
\begin{aligned}
R\left(z_{1}, \ldots, z_{n}\right)= & \frac{\int \varphi\left(z_{1}\right) \ldots \varphi\left(z_{n}\right) \exp \left\{-(1 / 2 \gamma) \int\left[\left(-\Delta+m^{2}\right) \varphi(z)+V^{\prime}(\varphi(z))\right]^{2} d z\right\}}{\int \exp \left\{-(1 / 2 \gamma) \int\left[\left(-\Delta+m^{2}\right) \varphi(z)+V^{\prime}(\varphi(z))\right]^{2} d z\right\}} \\
& \frac{\cdot \operatorname{det}\left(-\Delta+m^{2}+V^{\prime \prime}(\varphi)\right) \mathscr{D} \varphi}{\cdot \operatorname{det}\left(-\Delta+m^{2}+V^{\prime \prime}(\varphi)\right) \mathscr{D} \varphi}
\end{aligned}
$$

Even at the formal level there is need here to assume that $V$ is convex so (1.2) actually gives a change of variables [uniqueness of solutions of (1.2)] and the above determinant is positive so absolute values are not needed. (See Parisi and Sourlas [10] for a discussion of this problem.) But convexity is not needed if (1.5) is taken as a definition.

Parisi and Sourlas took (1.5) as the definition of $R\left(z_{1}, \ldots, z_{n}\right)$, re-expressed it as an expectation of a supersymmetric field theory, and explored the supersymmetry to show (1.4) order by order in perturbation theory.

We will now proceed to make their argument rigorous.

\section{Rigorous Results}

We will start by rewriting the right-hand side of (1.5) in a form more amenable to a rigorous treatment. By formal manipulations, we get

$$
R\left(z_{1}, \ldots, z_{n}\right)=\frac{\int \varphi\left(z_{1}\right) \ldots \varphi\left(z_{n}\right) \mathscr{H}(\varphi) d \mu(\varphi)}{\int \mathscr{H}(\varphi) d \mu(\varphi)},
$$

where

$$
\begin{aligned}
\mathscr{H}(\varphi)= & \exp \left\{-(1 / 2 \gamma) \int V^{\prime}(\varphi(z))^{2} d z-(1 / \gamma) \int\left(-\Delta+m^{2}\right) \varphi(z) V^{\prime}(\varphi(z)) d z\right\} \\
& \cdot \operatorname{det}\left(I+\left(-\Delta+m^{2}\right)^{-1 / 2} V^{\prime \prime}(\varphi)\left(-\Delta+m^{2}\right)^{-1 / 2}\right)
\end{aligned}
$$

and

$$
d \mu(\varphi)=\frac{\exp \left\{-(1 / 2 \gamma) \int\left[\left(-\Delta+m^{2}\right) \varphi(z)\right]^{2} d z\right\} \mathscr{D} \varphi}{\int \exp \left\{-(1 / 2 \gamma) \int\left[\left(-\Delta+m^{2}\right) \varphi(z)\right]^{2} d z\right\} \mathscr{D} \varphi} .
$$

The probability measure $d \mu(\varphi)$ can be defined rigorously as the probability distribution of the generalized Gaussian system $\left\{\varphi(z) ; z \in \mathbb{R}^{D}\right\}$ with mean zero and covariance $\left\langle\varphi(z) \varphi\left(z^{\prime}\right)\right\rangle=\gamma G_{2}\left(z-z^{\prime}\right)$, where $G_{s}(z)=(2 \pi)^{-D} \int e^{i q \cdot z}\left(q^{2}+m^{2}\right)^{-s} d q$ for $s \geqq 0$.

Notice that $G_{2}\left(z-z^{\prime}\right)$ is the Green's function for $\left(-\Delta+m^{2}\right)^{2}$.

The expression $\mathscr{H}(\varphi)$ does not make mathematical sense as it stands. To start we must introduce a space cutoff to handle the infinite volume integrations. Since the space cutoff is a variable coupling constant, the correct place to introduce it is in the definition of the Lagrangian [Eq. (1.1)]. So let $f$ be a positive function on $\mathbb{R}^{D}$, and let $\mathscr{L}(\varphi, f)=\frac{1}{2}(\nabla \varphi)^{2}+\frac{1}{2} m^{2} \varphi^{2}+f V(\varphi)$. Proceeding as before we get

$$
R\left(z_{1}, \ldots, z_{n} ; f\right)=\frac{\int \varphi\left(z_{1}\right) \ldots \varphi\left(z_{n}\right) \mathscr{H}(\varphi ; f) d \mu(\varphi)}{\int \mathscr{H}(\varphi ; f) d \mu(\varphi)},
$$


where

$$
\begin{aligned}
\mathscr{H}(\varphi ; f)= & \exp \left\{-(1 / 2 \gamma) \int V^{\prime}(\varphi(z))^{2} f(z)^{2} d z-(1 / \gamma) \int\left(-\Delta+m^{2}\right) \varphi(z) V^{\prime}(\varphi(z)) f(z) d z\right\} \\
& \cdot \operatorname{det}\left(I+\left(-\Delta+m^{2}\right)^{-1 / 2} f V^{\prime \prime}(\varphi)\left(-\Delta+m^{2}\right)^{-1 / 2}\right) .
\end{aligned}
$$

Here the determinant is taken in $L^{2}\left(\mathbb{R}^{D}, d z\right)$.

To define $\mathscr{H}(\varphi ; f)$ properly, we will need to impose certain conditions on the space cutoff $f$. But before we do that we will introduce a momentum cutoff in the first $D-2$ variables (in case $D \geqq 3$ ). So let $\chi(p)$ be a positive bounded integrable function in $\mathbb{R}^{D-2}$, and define $d \mu_{\chi}(\varphi)$ as the probability distribution of the generalized Gaussian system $\left\{\varphi(z) ; z \in \mathbb{R}^{D}\right\}$ with mean zero and covariance

$$
\left\langle\varphi(z) \varphi\left(z^{\prime}\right)\right\rangle=\gamma G_{2, \chi}\left(z-z^{\prime}\right),
$$

where

$$
G_{s, \chi}(z)=(2 \pi)^{-D} \int e^{i q \cdot z} \chi(p)\left(q^{2}+m^{2}\right)^{-s} d q
$$

for $s \geqq 0$. Recall $q=(p, k)$.

Notice that $G_{2, \chi}(0)$ is finite.

Let

$$
R_{\chi}\left(z_{1}, \ldots, z_{n} ; f\right)=\frac{\int \varphi\left(z_{1}\right) \ldots \varphi\left(z_{n}\right) \mathscr{H}_{\chi}(\varphi ; f) d \mu_{\chi}(\varphi)}{\int \mathscr{H}_{\chi}(\varphi ; f) d \mu_{\chi}(\varphi)}
$$

where

$$
\begin{aligned}
\mathscr{H}_{\chi}(\varphi ; f)= & \exp \left\{-(1 / 2 \gamma) \int V^{\prime}(\varphi(z)) V^{\prime}\left(\varphi\left(z^{\prime}\right)\right) f(z) f\left(z^{\prime}\right) G_{0, \chi}\left(z-z^{\prime}\right) d z d z^{\prime}\right. \\
& \left.-(1 / \gamma) \int\left(-\Delta+m^{2}\right) \varphi(z) V^{\prime}(\varphi(z)) f(z) d z\right\} \\
& \cdot \operatorname{det}\left(I+G_{1 / 2, \chi^{1 / 2}} f V^{\prime \prime}(\varphi) G_{1 / 2, \chi^{1 / 2}}\right),
\end{aligned}
$$

where we denote by $G_{s, \chi}$ the operator in $L^{2}\left(\mathbb{R}^{D}, d z\right)$ with kernel $G_{s, \chi}\left(z-z^{\prime}\right)$ given by (2.2). In this notation $G_{1 / 2,1}=\left(-\Delta+m^{2}\right)^{-1 / 2}$. Notice that $G_{1 / 2, \chi^{1 / 2}}^{2}=G_{1, \chi}$ as operators.

It will turn out that with the above definitions the first $D-2$ variables play no role in the dimensional reduction and we will be able to carry through the momentum cutoff $\chi$.

We will now give the technical definition for the space cutoff we will use. The definition will depend on a parameter $\delta$ that later will be taken to be the degree of the polynomial $V$ multiplied by the mass parameter $m^{2}$.

Definition. Let $\delta>0$. A $\delta$-appropriate space cutoff is a function $f: \mathbb{R}^{D} \rightarrow \mathbb{R}$ such that:

(i) $f \geqq 0$ of class $C^{2}$.

(ii) $f \in L^{1} \cap L^{2}\left(\mathbb{R}^{D}, d z\right)$.

(iii) $f(x, y)=\widetilde{f}\left(x, y^{2}\right)$ for some function $\tilde{f}: \mathbb{R}^{D-2} \times[0, \infty) \rightarrow \mathbb{R}$.

(iv) Let $\left.f^{\prime}(x, y) \equiv \tilde{f}^{\prime}\left(x, y^{2}\right) \equiv \frac{\partial}{\partial t} \tilde{f}(x, t)\right|_{t=y^{2}}$; then $f^{\prime} \leqq 0$ and $f^{\prime} \in L^{1}\left(\mathbb{R}^{D}, d z\right)$.

(v) $\Delta f \leqq b^{2} f$ with $b^{2}<\delta$ and $\Delta f \in L^{1}\left(\mathbb{R}^{D}, d z\right)$.

Notice that since $f$ is of class $C^{2}$ we must have $\tilde{f}$ of class $C^{1}$, hence $f^{\prime}$ is well defined and continuous. It follows that $f(\cdot, y) \in L^{1}\left(\mathbb{R}^{D-2}, d x\right)$ for all $y \in \mathbb{R}^{2}$. 
We will give examples of such functions. For $b>0,0<T_{1}<T_{2}$, let us define a function $\alpha_{b, T_{1}, T_{2}}:[0, \infty) \rightarrow[0, \infty)$ by $\alpha_{b, T_{1}, T_{2}}(t)=0$ for $0 \leqq t \leqq T_{1}$,

$$
\alpha_{b, T_{1}, T_{2}}(t)=\frac{b}{2}\left[t-T_{1}-\frac{T_{2}-T_{1}}{\pi} \cos \left\{\left(t-\frac{T_{1}+T_{2}}{2}\right) \frac{\pi}{T_{2}-T_{1}}\right\}\right]
$$

for $T_{1} \leqq t \leqq T_{2}$

$$
\alpha_{b, T_{1}, T_{2}}(t)=b\left[t-\frac{T_{1}+T_{2}}{2}\right] \text { for } T_{2} \leqq t .
$$

The following functions are $\delta$-appropriate space cutoffs:

$$
f(z)=\exp \left\{-\alpha_{b, T_{1}, T_{2}}(|z|)\right\}
$$

with $b^{2}<\delta$ and any $0<T_{1}<T_{2}$. Notice that $f(z)=1$ for $|z| \leqq T_{1}$ and $f(z)=c e^{-b|z|}$ for $|z| \geqq T_{2}$, with $c=\exp \left\{\frac{1}{2} b\left(T_{1}+T_{2}\right)\right\}$.

Notice that $f(z)=\widetilde{f}\left(z^{2}\right)$ for some function $\tilde{f}:[0, \infty) \rightarrow[0, \infty)$ and that in this case $f^{\prime}(z)=\tilde{f}^{\prime}\left(z^{2}\right)$, where $\tilde{f}^{\prime}$ has the usual meaning of the derivative of a function of one variable.

b)

$$
f(z)=\exp \left\{-\alpha_{b_{1}, X_{1}, X_{2}}(|x|)-\alpha_{b_{2}, Y_{1}, Y_{2}}(|y|)\right\},
$$

with $b_{1}^{2}+b_{2}^{2}<\delta$ and any $0<X_{1}<X_{2}, 0<Y_{1}<Y_{2}$.

As we mentioned before, the dimensional reduction will require a counterterm depending on the boundary of the space cutoff. We define

$$
\widetilde{\mathscr{H}}_{\chi}(\varphi ; f)=\exp \left\{(4 / \gamma) \int V(\varphi(z)) f^{\prime}(z) d z\right\} \mathscr{H}_{\chi}(\varphi ; f),
$$

and let $\widetilde{R}_{\chi}\left(z_{1}, \ldots, z_{m} ; f\right)$ be defined as in $(2.3)$ but with $\tilde{\mathscr{H}}_{\chi}(\varphi ; f)$ substituted for $\mathscr{H}_{\chi}(\varphi ; f)$.

We can now state

Theorem I. Let $V$ be a bounded below polynomial in one variable, and let $\delta$ be its degree. Let $f$ be a $m^{2} \delta$-appropriate space cutoff. Then $\mathscr{H}_{\chi}(\varphi ; f)$ and $\tilde{\mathscr{H}}_{\chi}(\varphi ; f)$ are in $L^{p}\left(d \mu_{\chi}(\varphi)\right)$ for all $1 \leqq p<\infty$.

If the polynomial $V$ is strictly convex (i.e., $V^{\prime \prime}>0$ ) and $f>0$, the determinant in (2.3) is strictly positive. Thus it follows from Theorem I that $R_{\chi}\left(z_{1}, \ldots, z_{n} ; f\right)$ and $\tilde{R}_{\chi}\left(z_{1}, \ldots, z_{n} ; f\right)$ are well defined since the denominators in the respective definitions are strictly positive and hence not zero.

But if $V$ is not strictly convex, there is no a priori reason for $\int \mathscr{H}_{\chi}(\varphi ; f) d \mu_{\chi}(\varphi)$ and $\int \tilde{\mathscr{H}}_{\chi}(\varphi ; f) d \mu_{\chi}(\varphi)$ to be different from zero, since $\mathscr{H}_{\chi}(\varphi ; f)$ can take on both positive and negative values. But it will follow from Theorem II that $\int \widetilde{\mathscr{H}}_{\chi}(\varphi, f) d \mu_{\chi}(\varphi)>0$, and hence that $\tilde{R}\left(z_{1}, \ldots, z_{n} ; f\right)$ is well defined.

We will now proceed to state the dimensional reduction. Let $\left\{\phi(x) ; x \in \mathbb{R}^{D-2}\right\}$ be the generalized Gaussian system with mean zero and covariance $\left\langle\phi(x) \phi\left(x^{\prime}\right)\right\rangle$ $=(\gamma / 4 \pi) H_{1, \chi}\left(x-x^{\prime}\right)$, where

$$
H_{r, \chi}(x)=(2 \pi)^{-(D-2)} \int e^{i p \cdot x} \chi(p)\left(p^{2}+m^{2}\right)^{-r} d p
$$

for $r \geqq 0$. Notice that $H_{1}\left(x-x^{\prime}\right)$ (without the cutoff $\chi$ ) is the Green's function of $-\Delta+m^{2}$ on $\mathbb{R}^{D-2}$. We will denote by $d v_{\chi}(\phi)$ the probability distribution of the field $\phi$. 
If $g \geqq 0, g \in L^{1}\left(\mathbb{R}^{D-2}, d x\right), \int V(\phi(x)) g(x) d x$ is well defined in $L^{2}\left(d v_{x}(\phi)\right)$ and $\exp \left\{-(4 \pi / \gamma) \int V(\phi(x)) g(x) d x\right\}$ is bounded (e.g., [11,12]). The cutoff Schwinger functions for $\phi$ are then well defined by

$$
S_{\chi}\left(x_{1}, \ldots, x_{n} ; g\right)=\frac{\int \phi\left(x_{1}\right) \ldots \phi\left(x_{n}\right) \exp \left\{-(4 \pi / \gamma) \int V(\phi(x)) g(x) d x\right\} d v_{\chi}(\phi)}{\int \exp \left\{-(4 \pi / \gamma) \int V(\phi(x)) g(x) d x\right\} d v_{\chi}(\phi)} .
$$

The dimensional reduction is given by

Theorem II. With the hypothesis of Theorem I, we have

$$
\begin{aligned}
& \int \varphi\left(x_{1}, 0\right) \ldots \varphi\left(x_{n}, 0\right) \tilde{\mathscr{H}}_{x}(\varphi ; f) d \mu_{\chi}(\varphi) \\
& \quad=\int \phi\left(x_{1}\right) \ldots \phi\left(x_{n}\right) \exp \left\{-(4 \pi / \gamma) \int V(\phi(x)) f(x, 0) d x\right\} d v_{\chi}(\phi)
\end{aligned}
$$

for all $x_{1}, \ldots, x_{n} \in \mathbb{R}^{D-2}$.

In particular,

$$
\tilde{R}_{\chi}\left(\left(x_{1}, 0\right), \ldots,\left(x_{n}, 0\right) ; f\right)=S_{\chi}\left(x_{1}, \ldots, x_{n} ; f(\cdot, 0)\right)
$$

for all $x_{1}, \ldots, x_{n} \in \mathbb{R}^{D-2}$.

\section{Proof of Theorem I}

We can write

$$
\begin{gathered}
\mathscr{H}_{\chi}(\varphi ; f)=\mathscr{H}_{1}(\varphi ; f) \mathscr{H}_{2}(\varphi ; f) \mathscr{H}_{3, \chi}(\varphi ; f), \\
\widetilde{\mathscr{H}}_{\chi}(\varphi ; f)=\mathscr{H}_{\chi}(\varphi ; f) \mathscr{H}_{4}(\varphi ; f),
\end{gathered}
$$

where

$$
\begin{gathered}
\mathscr{H}_{1, \chi}(\varphi ; f)=\exp \left\{-(1 / 2 \gamma) \int V^{\prime}(\varphi(z)) V^{\prime}\left(\varphi\left(z^{\prime}\right)\right) f(z) f\left(z^{\prime}\right) G_{0, \chi}\left(z-z^{\prime}\right) d z d z^{\prime}\right\}, \\
\mathscr{H}_{2}(\varphi ; f)=\exp \left\{-(1 / \gamma) \int\left(-\Delta+m^{2}\right) \varphi(z) V^{\prime}(\varphi(z)) f(z) d z\right\}, \\
\mathscr{H}_{3, \chi}(\varphi ; f)=\operatorname{det}\left(I+G_{1 / 2, \chi^{1 / 2}} f V^{\prime \prime}(\varphi) G_{1 / 2, \chi^{1 / 2}}\right), \\
\mathscr{H}_{4}(\varphi ; f)=\exp \left\{(4 / \gamma) \int V(\varphi(z)) f^{\prime}(z) d z\right\} .
\end{gathered}
$$

Since $\left\langle\varphi(z) \varphi\left(z^{\prime}\right)\right\rangle$ is bounded as $\left\langle\varphi(z)^{2}\right\rangle<\infty$, the exponents in $\mathscr{H}_{1, \chi}$ and $\mathscr{H}_{4}$ are well defined in $L^{2}\left(d \mu_{\chi}(\varphi)\right)$ for $f \in L^{2}$ and $f^{\prime} \in L^{1}$, respectively. Clearly $\mathscr{H}_{1, \chi}(\varphi ; f) \leqq 1$; and $\mathscr{H}_{4}(\varphi ; f)$ is bounded from above since $f^{\prime} \leqq 0, f^{\prime} \in L^{1}$, and $V$ is bounded below.

Thus we need only show that $\mathscr{H}_{2}(\varphi ; f) \mathscr{H}_{3, \chi}(\varphi ; f) \in L^{p}\left(d \mu_{\chi}(\varphi)\right)$ for all $1 \leqq p<\infty$. It will turn out that $\mathscr{H}_{2}$ and $\mathscr{H}_{3}$ cannot be defined as they stand, but their product $\mathscr{H}_{2} \mathscr{H}_{3, \chi}$ makes sense thanks to a cancellation.

Let us start with $\mathscr{H}_{2}(\varphi ; f)$. The problem in defining the exponent in the definition of $\mathscr{H}_{2}$ is that

$$
\left\langle\left(-\Delta+m^{2}\right) \varphi(z) \varphi\left(z^{\prime}\right)\right\rangle=\gamma G_{1, \chi}\left(z-z^{\prime}\right),
$$

and $G_{1, \chi}(z)$ has a logarithmic singularity at $z=0$. But it can be handled in the same way as Wick powers of the usual Euclidean scalar field in two dimensions (e.g., $[11,12])$. We do not need a full Wick ordering since $\left\langle\varphi(z) \varphi\left(z^{\prime}\right)\right\rangle$ has no singularities. 
So we define

$$
\mathscr{B}(\varphi ; f)=\int\left[\left(-\Delta+m^{2}\right) \varphi(z) V^{\prime}(\varphi(z))-\gamma G_{1, \chi}(0) V^{\prime \prime}(\varphi(z))\right] f(z) d z .
$$

Then

$$
\begin{aligned}
\left\langle\mathscr{B}(\varphi ; f)^{2}\right\rangle= & \iint\left[\gamma G_{0, \chi}\left(z-z^{\prime}\right)\left\langle V^{\prime}(\varphi(z)) V^{\prime}\left(\varphi\left(z^{\prime}\right)\right)\right\rangle\right. \\
& \left.+\gamma^{2} G_{1, \chi}\left(z-z^{\prime}\right)^{2}\left\langle V^{\prime \prime}(\varphi(z)) V^{\prime \prime}\left(\varphi\left(z^{\prime}\right)\right)\right\rangle\right] f(z) f\left(z^{\prime}\right) d z d z^{\prime}
\end{aligned}
$$

is finite for $f \in L^{2}$. Here $\left\langle>\right.$ is the expectation with respect to the measure $d \mu_{\chi}(\varphi)$.

Thus $\mathscr{B}(\varphi ; f)$ is well defined in $L^{2}\left(d \mu_{\chi}(\varphi)\right)$. All steps above and in what follows can be justified by introducing a momentum cutoff for the $y$ variables; the singularity of $G_{1, \chi}(z)$ at $z=0$ will be logarithmic in the cutoff. We will keep on supressing this extra cutoff for the remainder of this proof.

We now define

$$
\widetilde{\mathscr{H}}_{2, \chi}(\varphi ; f)=\exp \{-(1 / \gamma) \mathscr{B}(\varphi ; f)\} \text {. }
$$

We will show that $\tilde{\mathscr{H}}_{2, \chi}(\varphi ; f) \in L^{p}\left(d \mu_{\chi}(\varphi)\right)$ for all $1 \leqq p<\infty$.

Since $V$ is a bounded below polynomial, we can find $c, d \geqq 0$ such that $W(t)$ $=V(t)+(c / 2) t^{2}+d$ is convex $\left(W^{\prime \prime} \geqq 0\right)$ and positive $(W \geqq 0)$.

For a polynomial $P$ let us define $\mathscr{B}(\varphi ; f ; P)$ by $(3.1)$ with $P$ substituted for $V$, and let

$$
\mathscr{B}(\varphi ; f ; P ; \lambda)=\mathscr{B}(\varphi ; f ; P)-\lambda \int \varphi(z)^{2} f(z) d z
$$

Then

$$
\mathscr{B}(\varphi ; f)=\mathscr{B}(\varphi ; f ; V ; 0)=\mathscr{B}(\varphi ; f ; W ; \lambda)-\mathscr{B}\left(\varphi ; f ;(c / 2) t^{2} ; \lambda\right),
$$

for any $\lambda \in \mathbb{R}$, where $t^{2}$ denotes the polynomial $P\left(t^{2}\right)=t^{2}$. The constant $\lambda$ will be chosen later.

Lemma 3.1. $\exp \{-p \mathscr{B}(\varphi ; f ; W, \lambda)\} \in L^{1}\left(d \mu_{\chi}(\varphi)\right)$ for any $p>0$ and any $\lambda \in \mathbb{R}$.

Proof. Since the singularity in $G_{1, \chi}$ is logarithmic, and this is the only singularity, we can apply Nelson's method (e.g. $[11,12]$ ) so all we need is a bound of the form $\mathscr{B}(\varphi ; f ; W ; \lambda) \geqq-C_{1} G_{1, \chi}(0)^{C_{2}} \int f(z) d z$ for some constants $C_{1}, C_{2} \geqq 0$.

Now,

$$
\begin{aligned}
\int(-\Delta \varphi)(z) W^{\prime}(\varphi(z)) f(z) d z & =\int W^{\prime \prime}(\varphi(z))|\nabla \varphi(z)|^{2} f(z) d z+\int W(\varphi(z))(-\Delta f)(z) d z \\
& \geqq \int W(\varphi(z))(-\Delta f)(z) d z,
\end{aligned}
$$

since $W^{\prime \prime} \geqq 0$. Here we use the requirement that $\Delta f \in L^{1}$. But we also required that $\Delta f \leqq b^{2} f$, where $b^{2}<m^{2} \delta$. Thus, as $W \geqq 0$,

$$
\int(-\Delta \varphi)(z) W^{\prime}(\varphi(z)) f(z) d z \geqq-b^{2} \int W(\varphi(z)) f(z) d z .
$$

Thus

$$
\begin{aligned}
& \mathscr{B}(\varphi ; f ; W ; \lambda) \\
& \quad \geqq \int\left[m^{2} \varphi(z) W^{\prime}(\varphi(z))-b^{2} W(\varphi(z))-\gamma G_{1, \chi}(0) W^{\prime \prime}(\varphi(z))-\lambda \varphi^{2}(z)\right] f(z) d z \\
& \quad \geqq-C_{1} G_{1, \chi}(0)^{C_{2}} \int f(z) d z
\end{aligned}
$$

for some constants $C_{1}, C_{2} \geqq 0$, since we have $b^{2}<m^{2} \delta$. 
Lemma 3.2. Given $p>0$ there exists $\lambda>0$ such that $\exp \left\{p \mathscr{B}\left(\varphi ; f ; t^{2} ; \lambda\right)\right\}$ $\in L^{1}\left(d \mu_{\chi}(\varphi)\right)$.

Proof. We must show that given $p>0$ there exists $\lambda>0$ such that

$$
\exp \left\{p \int: \varphi(z)(K \varphi)(z): d z\right\} \in L^{1}\left(d \mu_{\chi}(\varphi)\right),
$$

where

$$
\begin{gathered}
K=\left(-\Delta+m^{2}\right) f+f\left(-\Delta+m^{2}\right)-\lambda f, \\
: \varphi(z)(K \varphi)(z):=\varphi(z)(K \varphi)(z)-\langle\varphi(z)(K \varphi)(z)\rangle .
\end{gathered}
$$

Here $f$ denotes the operator multiplication by $f$. Notice that since $\left\langle\varphi(0)^{2}\right\rangle<\infty$ we only added a finite constant.

Since $d \mu_{\chi}(\varphi)$ is the Gaussian measure with covariance $\gamma G_{2, \chi}\left(z-z^{\prime}\right)$, it suffices to show (e.g., [11])

(i) $\left(-\Delta+m^{2}\right)^{2}-2 \gamma p K_{\chi} \geqq \eta>0$ for some $\eta>0$ and $\lambda>0$;

(ii) $\left(-\Delta+m^{2}\right)^{-1} K_{\chi}\left(-\Delta+m^{2}\right)^{-1}$ is Hilbert-Schmidt for all $\lambda$;

we use the notation $\hat{A}$ to denote the operator $A$ acting on momentum space (i.e., $\hat{A}=\mathscr{J}^{-1} A \mathscr{J}, \mathscr{J}$ being the Fourier transform), and define $A_{\chi}$ by $\hat{A}_{\chi}=\chi^{1 / 2} \hat{A} \chi^{1 / 2}$.

To show (ii), let $A=\left(-\Delta+m^{2}\right)^{-1} K_{\chi}\left(-\Delta+m^{2}\right)^{-1}$. Then $\hat{A}$ has the kernel

$$
\begin{aligned}
\hat{A}\left(q, q^{\prime}\right)= & \chi(p)^{1 / 2}\left(q^{2}+m^{2}\right)^{-1}\left[\left(q^{2}+m^{2}\right)+\left(q^{\prime 2}+m^{2}\right)-\lambda\right] \\
& \cdot \hat{f}\left(q-q^{\prime}\right) \chi\left(p^{\prime}\right)^{1 / 2}\left(q^{\prime 2}+m^{2}\right)^{-1} .
\end{aligned}
$$

Since $\hat{A}\left(q, q^{\prime}\right)$ is square integrable, $A$ is Hilbert-Schmidt.

Let us now prove (i). Since $\chi$ is bounded, $\left[\left(-\Delta+m^{2}\right)\right]_{\chi} \leqq C\left(-\Delta+m^{2}\right)^{2}$ for some constant $C$. We also have $\left(-\Delta+m^{2}\right)^{2} \geqq m^{4}>0$. Thus it suffices to show that given $a>0,\left(-\Delta+m^{2}\right)^{2}-a K \geqq 0$ for some $\lambda>0$.

Let $B=\left(-\Delta+m^{2}\right)$. We must show $B^{2}-a[B f+f B-\lambda f] \geqq 0$. Now, for any $g$ in the domain of $B$ and $\varepsilon>0$,

$$
\left|\int(B g)(z) \tilde{g}(z) f(z) d z\right| \leqq(\varepsilon / 2) \int|(B g)(z)|^{2} f(z) d z+(1 / 2 \varepsilon) \int|g(z)|^{2} f(z) d z .
$$

Hence $B f+f B \geqq-\varepsilon B f B-(1 / \varepsilon) f$. Thus

$$
\begin{aligned}
B^{2}-a K & \geqq B^{2}-a \varepsilon B f B+a(\lambda-(1 / \varepsilon)) f \\
& =B(1-a \varepsilon f) B+a(\lambda-(1 / \varepsilon)) f .
\end{aligned}
$$

Since $f$ is bounded and positive, we can choose $\varepsilon>0$ such that $1-a \varepsilon f \geqq \frac{1}{2}$. Then we choose $\lambda$ such that $\lambda>(1 / \varepsilon)$. Then

$$
B^{2}-a K \geqq(1 / 2) B^{2} \geqq(1 / 2) m^{4}>0 .
$$

Thus $\tilde{\mathscr{H}}_{2, \chi}(\varphi ; f) \in L^{p}\left(d \mu_{\chi}(\varphi)\right)$ for all $1 \leqq p<\infty$. To finish the proof of Theorem I we will now show that

$$
\widetilde{\mathscr{H}}_{3, \chi}(\varphi ; f)=\mathscr{H}_{3, \chi}(\varphi ; f) \exp \left\{-G_{1, \chi}(0) \int V^{\prime \prime}(\varphi(z)) f(z) d z\right\}
$$

is well defined and in $L^{\infty}\left(d \mu_{\chi}(\varphi)\right)$.

Recall that

$$
\mathscr{H}_{3, \chi}(\varphi ; f)=\operatorname{det}\left(I+G_{1 / 2, \chi^{1 / 2}} f V^{\prime \prime}(\varphi) G_{1 / 2, \chi^{1 / 2}}\right) .
$$


It is not hard to show that

$$
\operatorname{Tr}\left(G_{1 / 2, \chi^{1 / 2}} f V^{\prime \prime}(\varphi) G_{1 / 2, \chi^{1 / 2}}\right)=G_{1, \chi}(0) \int V^{\prime \prime}(\varphi(z)) f(z) d z .
$$

Hence

$$
\tilde{\mathscr{H}}_{3, \chi}(\varphi ; f)=\operatorname{det}_{1}\left(I+G_{1 / 2, \chi^{1 / 2}} f V^{\prime \prime}(\varphi) G_{1 / 2, \chi^{1 / 2}}\right),
$$

where $\operatorname{det}_{1}(I+A)=\operatorname{det}\left((I+A) e^{-A}\right)$ is well defined for $A$ Hilbert-Schmidt. For $A$ trace class, $\operatorname{det}_{1}(I+A)=\operatorname{det}(I+A) e^{-T_{r} A}$.

Now, if $A=G_{1 / 2, \chi^{1 / 2}} g G_{1 / 2, \chi^{1 / 2}}$, its Hilbert-Schmidt norm can be computed:

$$
\begin{aligned}
\|A\|_{2}^{2} & =\int \chi(p)\left(q^{2}+m^{2}\right)^{-1}\left|\hat{g}\left(q-q^{\prime}\right)\right|^{2} \chi\left(p^{\prime}\right)\left(q^{\prime 2}+m^{2}\right)^{-1} d q d q^{\prime} \\
& \leqq\left\|\chi(p)\left(q^{2}+m^{2}\right)^{-1}\right\|_{2}^{2}\|g\|_{2}^{2}<\infty
\end{aligned}
$$

if $g \in L^{2}\left(\mathbb{R}^{D}, d z\right)$.

Since

$$
\int\left\langle V^{\prime \prime}(\varphi(z))^{2} f(z)^{2}\right\rangle d z=\left\langle V^{\prime \prime}(\varphi(0))^{2}\right\rangle \int f(z)^{2} d z,
$$

and $f \in L^{2}\left(\mathbb{R}^{D}, d z\right)$, we can conclude by Fubini's theorem that

$$
\int V^{\prime \prime}(\varphi(z))^{2} f(z)^{2} d z<\infty \text { for } \mu_{\chi}(\varphi) \text {-a.e. } \varphi,
$$

i.e., $V^{\prime \prime}(\varphi(z)) f^{\prime}(z) \in L^{2}\left(\mathbb{R}^{D}, d z\right)$ for $\mu_{\chi}(\varphi)$ - a.e. $\varphi$.

Hence $\widetilde{\mathscr{H}}_{3, \chi}(\varphi ; f)$ is well defined for $\mu_{\chi}(\varphi)$-a.e. $\varphi$.

To show $\mathscr{\mathscr { H }}_{3, \chi}(\varphi ; f) \in L^{\infty}$ we will need the following Lemma.

Lemma 3.3. Let $K, A, B$ be self-adjoint Hilbert-Schmidt operators, with $A$ and $B$ positive, such that $K=A-B$. Then

$$
\left|\operatorname{det}_{1}(I+K)\right| \leqq \exp \left\{2\|B\|_{2}^{2}\right\} .
$$

Proof. Let us assume first that $A$ and $B$ commute. Then it is easy to see that

$$
\operatorname{det}_{1}(1+K)=\operatorname{det}_{1}(I+A) \operatorname{det}_{1}(I-B) .
$$

Since $A \geqq 0,0<\operatorname{det}_{1}(I+A) \leqq 1$ as $0<(1+\lambda) e^{-\lambda} \leqq 1$ for $\lambda \geqq 0$.

Now let $\left\{\lambda_{i} ; i=1,2, \ldots\right\}$ be the non-zero eigenvalues of $B$, with multiplicity taken into account. Then

$$
\left|\operatorname{det}_{1}(I-B)\right|=\prod_{i=1}^{\infty}\left|\left(1-\lambda_{i}\right) e^{\lambda_{i}}\right| \leqq \exp \left[2 \sum_{i=1}^{\infty} \lambda_{i}^{2}\right]=e^{2\|B\|_{2}^{2}} .
$$

Now let us consider the general case. Let $P=\chi_{(-\infty, 0)}(K), \chi_{(-\infty, 0)}$ being the characteristic function of $(-\infty, 0)$, and let $K_{-}=-K P, K_{+}=K(1-P)$. Then $K_{+}, K_{-}$are positive, self-adjoint, Hilbert-Schmidt, $K=K_{+}-K_{-}$, and $K_{+}$and $K$ - commute. Hence $\left|\operatorname{det}_{1}(1+K)\right| \leqq e^{2\|K-\|_{2}^{2}}$.

Since $K=A-B$, we have that $K_{-}=-P K P \leqq P B P$ as $P A P \geqq 0$. Using $\|P B P\|_{2} \leqq\|B\|_{2}$,

Lemma 3.4. Let $A, B$ be self-adjoint Hilbert-Schmidt operators such that $0 \leqq A \leqq B$. Then $\|A\|_{2} \leqq\|B\|_{2}$. 
Proof. $B=A+C$, where $C \geqq 0$, self-adjoint, Hilbert-Schmidt. Then $B^{2}=A^{2}$ $+A C+C A+C^{2}$, so $\|B\|_{2}^{2}=\operatorname{Tr} B^{2}=\operatorname{Tr} A^{2}+\operatorname{Tr} C^{2}+2 \operatorname{Tr} A C=\operatorname{Tr} A^{2}+\operatorname{Tr} C^{2}$ $+2 \operatorname{Tr}\left(C^{1 / 2} A C^{1 / 2}\right) \geqq \operatorname{Tr} A^{2}=\|A\|_{2}^{2}$.

We now apply Lemma 3.3 to $K=G_{1 / 2, \chi^{1 / 2}} V^{\prime \prime}(\varphi) f G_{1 / 2, \chi^{1 / 2}}$. Let $V_{+}^{\prime \prime}=V^{\prime \prime} \vee 0$, $V_{-}^{\prime \prime}=-\left(V^{\prime \prime} \wedge 0\right)$. Since $V$ is a bounded below polynomial, so is $V^{\prime \prime}$, hence $V_{-}^{\prime \prime}$ is bounded above.

Let $B(\varphi)=G_{1 / 2, \chi^{1 / 2}} V_{-}^{\prime \prime}(\varphi) f G_{1 / 2, \chi^{1 / 2}}$. Then, as in (3.2), we conclude that $\|B(\varphi)\|_{2}^{2}$ $\leqq M\|f\|_{2}^{2}$ for some constant $M<\infty$ independent of $\varphi$.

Thus Lemma 3.3 tells us that for $\mu_{\chi}(\varphi)$-a.e. $\varphi$,

$$
\left|\widetilde{\mathscr{H}}_{3, \chi}(\varphi ; f)\right| \leqq e^{\frac{1}{2} M\|f\|_{2}^{2}}
$$

independently of $\varphi$.

Thus $\widetilde{\mathscr{H}}_{3, \chi}(\varphi ; f) \in L^{\infty}\left(d \mu_{\chi}(\varphi)\right)$.

This concludes the proof of Theorem I.

\section{Supersymmetries: Some Basic Facts}

Supersymmetries are rotations of the "superspace" with "variables" $(z, \theta, \bar{\theta})$, where $z \in \mathbb{R}^{D}$ and $\theta$ and $\bar{\theta}$ are anticommuting "variables" (i.e., $\theta^{2}=\bar{\theta}^{2}=\theta \bar{\theta}+\bar{\theta} \theta=0$ ), which preserve the supermetric $z^{2}+(4 / \gamma) \bar{\theta} \theta$ (e.g., $[5,7]$ ).

In addition to the usual rotations in $\mathbb{R}^{D}$ and symplectic transformations of $\theta$ and $\bar{\theta}$, they include transformations of the type:

$$
\begin{aligned}
& z \rightarrow z+2 \bar{b} \xi \theta+2 b \xi \bar{\theta}, \\
& \theta \rightarrow \theta+\gamma b \cdot z \xi, \\
& \bar{\theta} \rightarrow \bar{\theta}-\gamma \bar{b} \cdot z \xi,
\end{aligned}
$$

where $b, \bar{b} \in \mathbb{R}^{D}$ and $\xi$ is an anticommuting "c-number" $\left(\xi^{2}=\xi \theta+\theta \xi=\xi \bar{\theta}\right.$ $+\bar{\theta} \xi=0)$. We will fix $\xi$ and call the above transformation $\tau(b, \bar{b})$.

One way to make the above discussion rigorous is to consider a Grassman algebra $\mathscr{G}_{2}$ with two generators $\theta$ and $\bar{\theta}$, and look at functions $F: \mathbb{R}^{D} \rightarrow \mathscr{G}_{2}$. Since $\mathscr{G}_{2}$ is a four-dimensional vector space with basis $1, \theta, \bar{\theta}$, and $\bar{\theta} \theta$, such a function can be written in a unique way as

$$
F(z)=F_{0}(z)+F_{1}(z) \theta+F_{2}(z) \bar{\theta}+F_{3}(z) \bar{\theta} \theta,
$$

where $F_{i}: \mathbb{R}^{D} \rightarrow \mathbb{C}$ for $i=0,1,2,3$. To emphasize that $F$ takes values in the Grassman algebra generated by $\theta$ and $\bar{\theta}$ it will be useful to use the notation $F(z, \theta, \bar{\theta})$. In this connection it is suggestive to think of $\theta$ and $\bar{\theta}$ as anticommuting variables.

To define the action of supersymmetries on such functions $F(z, \theta, \bar{\theta})$ we need to consider a bigger Grassman algebra $\mathscr{G}_{3}$ with three generators $\theta, \bar{\theta}$, and $\xi$, and $\operatorname{imbed} \mathscr{G}_{2}$ in $\mathscr{G}_{3}$ in the obvious way. If we now consider $H: \mathbb{R}^{D} \rightarrow \mathscr{G}_{3}$, we can always write $H(z)=F(z, \theta, \bar{\theta})+G(z, \theta, \bar{\theta}) \xi$ in a unique way, where $F, G: \mathbb{R}^{D} \rightarrow \mathscr{G}_{2}$.

We will say that $F: \mathbb{R}^{D} \rightarrow \mathscr{G}_{2}$ is of class $C^{1,0}$ if $F_{0}, F_{1}, F_{2}$ are of class $C^{1}$ and $F_{3}$ is of class $C^{0} ; H: \mathbb{R}^{D} \rightarrow \mathscr{G}_{3}$ is of class $C^{1,0}$ if both $F$ and $G$ are. 
The action of supersymmetries on function of class $C^{1,0}$ will now be defined. The action of space rotations and pure symplectic transformations is obvious. The action of the supersymmetry $\tau(b, \bar{b})$ can now be defined from (4.1) by

$$
g(z+2 \bar{b} \xi \theta+2 b \xi \bar{\theta})=g(z)+2 \nabla g(z) \cdot(\bar{b} \xi \theta+b \xi \bar{\theta}),
$$

where $g: \mathbb{R}^{D} \rightarrow \mathbb{C}$ is a function of class $C^{1}$. Thus for $F: \mathbb{R}^{D} \rightarrow \mathscr{G}_{2}$ of class $C^{1,0}$,

$$
\begin{aligned}
(\tau(b, \bar{b}) F)(z)= & F(z, \theta, \bar{\theta})+\left[\left(\gamma b \cdot z F_{1}(z)-\gamma \bar{b} \cdot z F_{2}(z)\right)\right. \\
& +\left(-2 \nabla F_{0}(z) \cdot \bar{b}+\gamma \bar{b} \cdot z F_{3}(z)\right) \theta+\left(-2 \nabla F_{0}(z) \cdot b+\gamma b \cdot z F_{3}(z)\right) \bar{\theta} \\
& \left.+2\left(\nabla F_{1}(z) \cdot b-\nabla F_{2}(z) \cdot \bar{b}\right) \bar{\theta} \theta\right] \xi .
\end{aligned}
$$

We will say that $F(z, \theta, \bar{\theta})$ is supersymmetric if it is left invariant by all supersymmetries.

Proposition. Let $F(z, \theta, \bar{\theta})$ be of class $C^{0,1}$. The following are equivalent:

(i) $F(z, \theta, \bar{\theta})$ is supersymmetric.

(ii) $F(z, \theta, \bar{\theta})$ is left invariant by $\tau(b, \bar{b})$ for all $b, \bar{b} \in \mathbb{R}^{D}$.

(iii) $F_{1}(z)=F_{2}(z)=0$, and

$$
(2 / \gamma) \nabla F_{0}(z)=z F_{3}(z) .
$$

(iv) There exists a function $f:[0, \infty) \rightarrow \mathbb{C}$ of class $C^{1}$ such that $F(z, \theta, \bar{\theta})$ $=f\left(z^{2}+(4 / \gamma) \bar{\theta} \theta\right) \equiv f\left(z^{2}\right)+(4 / \gamma) f^{\prime}\left(z^{2}\right) \bar{\theta} \theta$.

Proof. (ii) $\Rightarrow$ (iii):

If (ii) holds, the term in $\xi$ of the right-hand-side of (4.2) must be zero for all $b, \bar{b} \in \mathbb{R}^{D}$. We can immediately conclude (iii). (iii) $\Rightarrow$ (iv):

(4.3) implies that $F_{0}(z)$ is a function only of $|z|$, hence we can write $F_{0}(z)=f\left(z^{2}\right)$ for a function $f:[0, \infty) \rightarrow \mathbb{R}$. Since $F_{0}$ is of class $C^{1}$ and $F_{3}$ is of class $C^{0}$, it can be shown from (4.3) that $f$ is of class $C^{1}$ and $F_{3}(z)=(4 / \gamma) f^{\prime}\left(z^{2}\right)$.

Since (i) $\Rightarrow$ (ii) is obvious and (iv) $\Rightarrow$ (i) is easy to see this concludes the proof.

Following [13], we define integration over anticommuting variables by:

$$
\int F(z, \theta, \bar{\theta}) d \bar{\theta} d \theta=-F_{3}(z), \quad \int F(z, \theta, \bar{\theta}) \xi d \bar{\theta} d \theta=-F_{3}(z) \xi .
$$

Thus the integration is defined simply as a linear functional on $\mathscr{G}_{2}$ or $\mathscr{G}_{3}$.

We will say that $F(z, \theta, \bar{\theta})$ of class $C^{1,0}$ is integrable if $F_{0}, \nabla F_{0}, F_{1}, \nabla F_{1}, F_{2}$, $\nabla F_{2}, F_{3}$ are integrable. For such functions we can define

$$
\int F(z, \theta, \bar{\theta}) d \bar{\theta} d \theta d z=\int\left[\int F(z, \theta, \bar{\theta}) d \bar{\theta} d \theta\right] d z=\int\left[\int F(z, \theta, \bar{\theta}) d z\right] d \bar{\theta} d \theta .
$$

Proposition 4.2. The integration over $z, \theta, \bar{\theta}$ is supersymmetric, i.e., if $F(z, \theta, \bar{\theta})$ is integrable of class $C^{1,0}$, and $\tau$ is a supersymmetry, then

$$
\int \tau F(z, \theta, \bar{\theta}) d \bar{\theta} d \theta d z=\int F(z, \theta, \bar{\theta}) d \bar{\theta} d \theta d z .
$$

Proof. The only non-obvious case is when $\tau=\tau(b, \bar{b})$. Then

$$
\int F(z, \theta, \bar{\theta}) d \bar{\theta} d \theta d z=-\int F_{3}(z) d z,
$$


and

$$
\int \tau F(z, \theta, \bar{\theta}) d \bar{\theta} d \theta d z=-\int F_{3}(z) d z-2 \int\left[\nabla F_{1}(z) \cdot b-\nabla F_{2}(z) \cdot \bar{b}\right] d z \xi .
$$

But since $F_{i}$ of class $C^{1}, F_{i}, \nabla F_{i} \in L^{1}$, we have that

$$
\int \nabla F_{i}(z) d z=0 \text { for } i=1,2 \text {. }
$$

The following lemma gave Parisi and Sourlas the dimensional reduction order by order in perturbation theory. Notice that it explains why the reduction is of two dimensions.

Lemma 4.3 [5]. Let $F(y, \theta, \bar{\theta})$ be a supersymmetric integrable function of class $C^{1,0}$, where $y \in \mathbb{R}^{2}$. Then

$$
\int F(y, \theta, \bar{\theta}) d \bar{\theta} d \theta d y=(4 \pi / \gamma) F_{0}(0) .
$$

Proof. By Proposition 4.1,

$$
F(y, \theta, \bar{\theta})=f\left(y^{2}+(4 / \gamma) \bar{\theta} \theta\right),
$$

where $f:[0, \infty) \rightarrow \mathbb{C}$ of class $C^{1}$. Since $F$ is integrable and $F_{0}(y)=f\left(y^{2}\right)$, we have that $\lim _{t \rightarrow \infty} f(t)=0$.

Thus

$$
\begin{aligned}
\int F(y, \theta, \bar{\theta}) d \bar{\theta} d \theta d y & =-(4 / \gamma) \int f^{\prime}\left(y^{2}\right) d y=-(8 \pi / \gamma) \int_{0}^{\infty} f^{\prime}\left(r^{2}\right) r d r \\
& =(4 \pi / \gamma) f(0)=(4 \pi / \gamma) F_{0}(0) . \quad \square
\end{aligned}
$$

Remark. Let $z=(u, v)$, where $u \in \mathbb{R}^{D-k}, v \in \mathbb{R}^{k}$. The above analysis applies to functions $F(u, v, \theta, \bar{\theta})$ that are supersymmetric in the $v, \theta, \bar{\theta}$ variables. In particular Proposition 4.1 says if $F(u, v, \theta, \bar{\theta})$ is supersymmetric in $v, \theta, \bar{\theta}$, then $(2 / \gamma) \nabla_{v} F_{0}(z)$ $=v F_{3}(z)$, and there exists a function $f: \mathbb{R}^{D-k} \times[0, \infty) \rightarrow \mathbb{C}$ such that $F(u, v, \theta, \bar{\theta})$ $=f\left(u, v^{2}+(4 / \gamma) \bar{\theta} \theta\right)$.

Of particular interest for us will be the case $z=(x, y)$ with $x \in \mathbb{R}^{D-2}, y \in \mathbb{R}^{2}$, and the supersymmetry is in the $y, \theta, \bar{\theta}$ variables. In this case Lemma 4.3 tells us that if $F(x, y, \theta, \bar{\theta})$ is supersymmetric in $y, \theta, \bar{\theta}$, then

$$
\int F(x, y, \theta, \bar{\theta}) d \bar{\theta} d \theta d y d x=(4 \pi / \gamma) \int F_{0}(x, 0) d x .
$$

We will need an extension of the previous results to distributions. Distributions $T(z, \theta, \bar{\theta})$ can be defined in the usual way by the formal formula

$$
T(F)=\int T(z, \theta, \bar{\theta}) F(z, \theta, \bar{\theta}) d \bar{\theta} d \theta d z .
$$

Let us write

$$
T(z, \theta, \bar{\theta})=T_{0}(z)+T_{1}(z) \theta+T_{2}(z) \bar{\theta}+T_{3}(z) \theta \bar{\theta},
$$

where $T_{0}, T_{1}, T_{4}, T_{3}$ are distributions in $z$. Then

$$
T(F)=T_{3}\left(F_{0}\right)-T_{0}\left(F_{3}\right)+T_{1}\left(F_{2}\right)-T_{2}\left(F_{1}\right) .
$$

If $\tau$ is a supersymmetry, its action on distributions is defined in the usual way. We will say that $T(z, \theta, \bar{\theta})$ is a supersymmetric distribution if for all 
supersymmetries $\tau$ we have $T(\tau F)=T(F)$ for all functions $F(z, \theta, \bar{\theta})$ in the domain of definition of $T(z, \theta, \bar{\theta})$. Notice that

$$
\begin{aligned}
T(\tau(b, \bar{b}) F)= & T(F)+\left[T_{3}\left(\gamma b \cdot z F_{1}\right)-T_{3}\left(\gamma \bar{b} \cdot z F_{2}\right)\right. \\
& -2 T_{0}\left(\nabla F_{1} \cdot b\right)+2 T_{0}\left(\nabla F_{2} \cdot \bar{b}\right)-2 T_{1}\left(\nabla F_{0} \cdot b\right) \\
& \left.+T_{1}\left(\gamma b \cdot z F_{3}\right)+2 T_{2}\left(\nabla F_{0} \cdot \bar{b}\right)-T_{2}\left(\gamma \bar{b} \cdot z F_{3}\right)\right] \xi
\end{aligned}
$$

Proposition 4.4. A distribution $T(u, v, \theta, \bar{\theta})$ is supersymmetric in $v, \theta, \bar{\theta}$ if and only if

$$
T(u, v, \theta, \bar{\theta})=T_{0}(u, v)+T_{3}(u, v) \theta \bar{\theta},
$$

where $T_{0}(u, v)$ and $T_{3}(u, v)$ are distributions invariant under rotations in $v$ such that

$$
(2 / \gamma) \nabla_{v} T_{0}=-v T_{3}
$$

Proof. It follows from (4.4) that $T(u, v, \theta, \bar{\theta})$ is invariant under $\tau((0, b),(0, \bar{b}))$ for all $b, \bar{b} \in \mathbb{R}^{k}$ if and only if

$$
\begin{gathered}
T_{3}\left(b \cdot v F_{1}\right)=(2 / \gamma) T_{0}\left(\nabla_{v} F_{1}\right), \\
T_{1}\left(b \cdot v F_{3}\right)=T_{2}\left(\bar{b} \cdot v F_{3}\right), \\
T_{1}\left(\nabla_{v} F_{0} \cdot b\right)=T_{2}\left(\nabla_{v} F_{0} \cdot \bar{b}\right),
\end{gathered}
$$

for all $b, \bar{b} \in \mathbb{R}^{k}$ and all $F_{0}, F_{1}, F_{3}$ on $\mathbb{R}^{D}$.

Since $T(u, v, \theta, \bar{\theta})$ is invariant under symplectic transformations in $\theta, \bar{\theta}$ if and only if $T_{1}=T_{2}=0$, and is invariant under rotations in $v$ if and only if $T_{0}, T_{1}, T_{2}, T_{3}$ are, the Proposition follows.

We will now prove the extension of Lemma 4.3 to distributions. We will state it under the conditions we will actually use it, but more general conditions can be easily given.

Lemma 4.5. Let $T(x, y, \theta, \bar{\theta})$ be a distribution supersymmetric in $y, \theta, \bar{\theta}$, where $y \in \mathbb{R}^{2}$, such that

(i) $T_{0}(x, y)$ is a bounded continuous function.

(ii) $\left|T_{3}(g)\right| \leqq C\left(\|g\|_{1}+\|g\|_{2}\right)$ for some constant $C$ and all functions $g: \mathbb{R}^{D} \rightarrow \mathbb{C}$ of class $C^{1}$.

Then, if $F(x, y, \theta, \bar{\theta})$ is a function of class $C^{1,0}$, supersymmetric in $y, \theta, \bar{\theta}$, with $F_{0} \in L^{1} \cap L^{2}\left(\mathbb{R}^{D}, d z\right)$ and $F_{3} \in L^{1}\left(\mathbb{R}^{D}, d z\right)$, we have

$$
T(F)=(4 \pi / \gamma) \int T_{0}(x, 0) F_{0}(x, 0) d x .
$$

Proof. From Proposition 4.4 we have that $T(x, y, \theta, \bar{\theta})=T_{0}(x, y)+T_{3}(x, y) \theta \bar{\theta}$ with

$$
(2 / \gamma) T_{0}\left(\nabla_{y} g\right)=T_{3}(y g)
$$

for all functions $g$ on $\mathbb{R}^{D}$ of class $C^{1}$ such that $\nabla_{y} g \in L^{1}\left(\mathbb{R}^{D}, d z\right), y g \in L^{1} \cap L^{2}\left(\mathbb{R}^{D}, d z\right)$.

Since $F(x, y, \theta, \bar{\theta})$ is supersymmetric in $y, \theta, \bar{\theta}, \quad F(x, y, \theta, \bar{\theta})=F_{0}(x, y)$ $+F_{3}(x, y) \bar{\theta} \theta$ with

$$
(2 / \gamma) \nabla_{y} F_{0}(x, y)=y F_{3}(x, y)
$$

Let $\varepsilon>0$, we have

$$
\nabla_{y} \cdot y\left(y^{2}+\varepsilon\right)^{-1}=2 \varepsilon\left(y^{2}+\varepsilon\right)^{-2} .
$$


Thus

$$
\nabla_{y} \cdot y\left(y^{2}+\varepsilon\right)^{-1} F_{0}=2 \varepsilon\left(y^{2}+\varepsilon\right)^{-2} F_{0}+y\left(y^{2}+\varepsilon\right)^{-1} \cdot \nabla_{y} F_{0} .
$$

We now apply $T_{0}$ to both sides and use (4.5) to get

$$
T_{3}\left(y^{2}\left(y^{2}+\varepsilon\right)^{-1} F_{0}\right)=(4 / \gamma) T_{0}\left(\varepsilon\left(y^{2}+\varepsilon\right)^{-2} F_{0}\right)+(2 / \gamma) T_{0}\left(y\left(y^{2}+\varepsilon\right)^{-1} \cdot \nabla_{y} F_{0}\right) .
$$

Using (4.6) we get

$$
T_{3}\left(y^{2}\left(y^{2}+\varepsilon\right)^{-1} F_{0}\right)-T_{0}\left(y^{2}\left(y^{2}+\varepsilon\right)^{-1} F_{3}\right)=(4 / \gamma) T_{0}\left(\varepsilon\left(y^{2}+\varepsilon\right)^{-2} F_{0}\right) .
$$

Since as $\varepsilon \rightarrow 0 y^{2}\left(y^{2}+\varepsilon\right)^{-1} F_{0} \rightarrow F_{0}$ in $L^{1} \cap L^{2}$ and $y^{2}\left(y^{2}+\varepsilon\right)^{-1} F_{3} \rightarrow F_{3}$ in $L^{1}$, we can conclude from (i) and (ii) that

$$
T(F)=T_{3}\left(F_{0}\right)-T_{0}\left(F_{3}\right)=(4 / \gamma) \lim _{\varepsilon \rightarrow 0} T_{0}\left(\varepsilon\left(y^{2}+\varepsilon\right)^{-2} F_{0}\right) .
$$

But

$$
\begin{aligned}
T_{0}\left(\varepsilon\left(y^{2}+\varepsilon\right)^{-2} F_{0}\right) & =\int T_{0}(x, y) \varepsilon\left(y^{2}+\varepsilon\right)^{-2} F_{0}(x, y) d x d y \\
& =\int \varepsilon\left(y^{2}+\varepsilon\right)^{-2} h(y) d y,
\end{aligned}
$$

where $h(y)=\int T_{0}(x, y) F_{0}(x, y) d x$.

By Fubini, $h(y)$ is defined a.e. But since $F_{0}$ is of class $C^{1}$ with (4.6) holding with $F_{3}$ of class $C^{0}$, it follows that $F_{0}(\cdot, y) \in L^{1}\left(\mathbb{R}^{D-2}, d x\right)$ for all $y \in \mathbb{R}^{2}$ and $h(y)$ is a continuous function.

Since $\varepsilon\left(y^{2}+\varepsilon\right)^{-2} \rightarrow \pi \delta(y)$ in $\mathbb{R}^{2}$ as $\varepsilon \rightarrow 0$ and $h(y)$ is a continuous function, we conclude that

$$
\lim _{\varepsilon \rightarrow 0} \int \varepsilon\left(y^{2}+\varepsilon\right)^{-2} h(y) d y=\pi h(0)
$$

Thus

$$
T(F)=(4 / \gamma) \int T_{0}(x, 0) F_{0}(x, 0) d x
$$

\section{Supersymmetric Free Field Theories}

Let us consider an Euclidean free field $\varphi(z)$ in $D$ dimensions with two-point function (i.e., covariance) $\left\langle\varphi(z) \varphi\left(z^{\prime}\right)\right\rangle=\gamma S\left(z-z^{\prime}\right)$, where $\gamma>0$.

$S(z)$ is a rotation invariant tempered distribution which we will formally write as

$$
S(z)=S\left(z^{2}\right)
$$

Recall that the $n$-point functions are given by (e.g., $[11,12])$

$$
\left\langle\varphi\left(z_{1}\right) \ldots \varphi\left(z_{n}\right)\right\rangle=\gamma^{(n / 2)} S_{n}\left(z_{1}, \ldots, z_{n}\right),
$$

where

$$
\begin{array}{ll}
S_{n}\left(z_{1}, \ldots, z_{n}\right)=0 & \text { for } n \text { odd } \\
S_{n}\left(z_{1}, \ldots, z_{n}\right)=\sum S\left(z_{i_{1}}-z_{j_{1}}\right) \ldots S\left(z_{i_{m}}-z_{j_{m}}\right) & \text { for } \quad n=2 m
\end{array}
$$


where the sum is over all possible ways of writing $1,2, \ldots, 2 m$ as $m$ distinct (unordered) pairs $\left(i_{1}, j_{1}\right), \ldots,\left(i_{m}, j_{m}\right)$. Thus

$$
\left\langle\varphi\left(z_{1}\right) \ldots \varphi\left(z_{n}\right)\right\rangle=\gamma^{(n / 2)} s_{n}\left(\left(z_{i}-z_{j}\right)^{2} ; i, j=1, \ldots, n, i<j\right),
$$

where $s_{n}$ is defined by (5.2) and (5.1).

A supersymmetric free field $\Phi(z, \theta, \bar{\theta})$ will be defined by the requirement that its $n$-point functions be supersymmetric counterparts of (5.3), i.e.,

$$
\begin{aligned}
& \left\langle\Phi\left(z_{1}, \theta_{1}, \bar{\theta}_{1}\right) \ldots \Phi\left(z_{n}, \theta_{n}, \bar{\theta}_{n}\right)\right\rangle \\
& \quad=\gamma^{(n / 2)} s_{n}\left(\left(z_{i}-z_{j}\right)^{2}+(4 / \gamma)\left(\bar{\theta}_{i}-\bar{\theta}_{j}\right)\left(\theta_{i}-\theta_{j}\right) ; i, j=1, \ldots, n, i<j\right),
\end{aligned}
$$

where $\left\{\bar{\theta}_{i}, \theta_{i} ; i=1, \ldots, n\right\}$ are independent anticommuting variables.

In particular, we must have

$$
\left\langle\Phi(z, \theta, \bar{\theta}) \Phi\left(z^{\prime}, \theta^{\prime}, \bar{\theta}^{\prime}\right)\right\rangle=\gamma s\left(\left(z-z^{\prime}\right)^{2}+(4 / \gamma)\left(\bar{\theta}-\bar{\theta}^{\prime}\right)\left(\theta-\theta^{\prime}\right)\right) .
$$

If we write

$$
\Phi(z, \theta, \bar{\theta})=\varphi(z)+\bar{\psi}(z) \theta+\bar{\theta} \psi(z)+\omega(z) \theta \bar{\theta}
$$

(5.5) means

$$
\begin{gathered}
\left\langle\varphi(z) \varphi\left(z^{\prime}\right)\right\rangle=\gamma s\left(\left(z-z^{\prime}\right)^{2}\right), \\
\left\langle\varphi(z) \psi\left(z^{\prime}\right)\right\rangle=\left\langle\varphi(z) \bar{\psi}\left(z^{\prime}\right)\right\rangle=\left\langle\psi(z) \varphi\left(z^{\prime}\right)\right\rangle=\left\langle\bar{\psi}(z) \varphi\left(z^{\prime}\right)\right\rangle=0, \\
\left\langle\bar{\psi}(z) \psi\left(z^{\prime}\right)\right\rangle=-\left\langle\psi(z) \bar{\psi}\left(z^{\prime}\right)\right\rangle=4 s^{\prime}\left(\left(z-z^{\prime}\right)^{2}\right), \\
\left\langle\psi(z) \psi\left(z^{\prime}\right)\right\rangle=\left\langle\bar{\psi}(z) \bar{\psi}\left(z^{\prime}\right)\right\rangle=0, \\
\left\langle\omega(z) \omega\left(z^{\prime}\right)\right\rangle=0, \\
\left\langle\varphi(z) \omega\left(z^{\prime}\right)\right\rangle=\left\langle\omega(z) \varphi\left(z^{\prime}\right)\right\rangle=-4 s^{\prime}\left(\left(z-z^{\prime}\right)^{2}\right), \\
\left\langle\psi(z) \omega\left(z^{\prime}\right)\right\rangle=\left\langle\omega(z) \psi\left(z^{\prime}\right)\right\rangle=\left\langle\bar{\psi}(z) \omega\left(z^{\prime}\right)\right\rangle=\left\langle\omega(z) \bar{\psi}\left(z^{\prime}\right)\right\rangle=0 .
\end{gathered}
$$

To satisfy (5.7)-(5.13) we may take $\varphi$ and $\omega$ to be commuting fields and $\psi, \bar{\psi}$ to be anticommuting fields.

Before we can give a rigorous construction for the superfield $\Phi(z, \theta, \bar{\theta})$ by (5.6) and (5.7)-(5.13) we must explain what we mean by $S(z, \theta, \bar{\theta})=s\left(z^{2}+(4 / \gamma) \bar{\theta} \theta\right)$, since $s\left(z^{2}\right)=S(z)$ is only a tempered distribution. What we need is a tempered distribution $S(z, \theta, \bar{\theta})$ which is a supersymmetric counterpart to $S(z)$. By Proposition $4.4 S(z, \theta, \bar{\theta})$ will have to be of the form $S(z, \theta, \bar{\theta})=S(z)+(4 / \gamma) T(z) \theta \bar{\theta}$, where $T(z)$ is a rotation invariant tempered distribution such that

$$
\nabla S=-2 z T \text {. }
$$

But a solution to (5.14) is not unique since $z T=0$ as tempered distributions only implies that $T$ is proportional to a delta function at zero.

In addition, the construction of the superfield requires a positive definite solution for (5.14). The following lemma gives sufficient conditions.

Lemma 5.1. Let $S(z)=\int e^{i q \cdot z} h\left(q^{2}\right) d q$, where $h$ is a positive measurable function on $[0, \infty)$ such that $\int_{0}^{\infty} h(r) d r<\infty$. Then $(5.14)$ has a positive definite solution $S_{1}(z)$ 
given by

$$
S_{1}(z)=\left(\frac{1}{4}\right) \int e^{i q \cdot z} g\left(q^{2}\right) d q,
$$

where

$$
g(r)=\int_{r}^{\infty} h\left(r^{\prime}\right) d r^{\prime} \quad \text { for } \quad r \geqq 0 .
$$

Proof. Notice that $g$ is an absolutely continuous bounded positive function on $[0, \infty)$ such that $g^{\prime}(r)=-h(r)$ a.e. Thus

$$
\nabla_{q} g\left(q^{2}\right)=2 q g^{\prime}\left(q^{2}\right)=-2 q h\left(q^{2}\right) \text { a.e. }
$$

As tempered distributions,

$$
\begin{aligned}
\nabla_{z} S(z) & =\int e^{i q \cdot z} i q h\left(q^{2}\right) d q \\
& =-(i / 2) \int e^{i q \cdot z} \nabla_{q} g\left(q^{2}\right) d q=-(1 / 2) z \int e^{i q \cdot z} g\left(q^{2}\right) d q .
\end{aligned}
$$

Thus

$$
S_{1}(z)=\frac{1}{4} \int e^{i q \cdot z} g\left(q^{2}\right) d q
$$

is the desired positive definite solution to (5.14).

We will now assume the hypothesis of the lemma and proceed to construct the superfield. We define $s^{\prime}\left(z^{2}\right)=-S_{1}(z)$. By (5.7) we are justified in taking the original Euclidean free field $\varphi(z)$ as the scalar part of the superfield.

To satisfy (5.10) we take

$$
\omega(z)=\varrho(z)+i \eta(z),
$$

where $\varrho(z), \eta(z)$ are identically distributed independent Gaussian generalized processes; i.e., $\varrho(z), \eta(z)$ are Gaussian generalized processes will mean zero such that $\left\langle\varrho(z) \eta\left(z^{\prime}\right)\right\rangle=0$ and $\left\langle\varrho(z) \varrho\left(z^{\prime}\right)\right\rangle=\left\langle\eta(z) \eta\left(z^{\prime}\right)\right\rangle$.

Equation (5.12) tells us that $\eta$ should be independent of $\varphi$, but

$$
\left\langle\varphi(z) \varrho\left(z^{\prime}\right)\right\rangle=-4 s^{\prime}\left(\left(z-z^{\prime}\right)^{2}\right) .
$$

From Lemma 5.1, if $s\left(z^{2}\right)=\int e^{i q \cdot z} h\left(q^{2}\right) d q$, then $-4 s^{\prime}\left(z^{2}\right)=\int e^{i q \cdot z} g\left(q^{2}\right) d q$, where $g(r)=\int_{r}^{\infty} h\left(r^{\prime}\right) d r^{\prime}$. Thus $(5.15)$ is satisfied if we take $\varrho(z)=(1 / \gamma) l(-\Delta) \varphi(z)$, where $l(r)$ is a function on $[0, \infty)$ such that $l(r) h(r)=g(r)$.

In this case $\left\langle\varrho(z) \varrho\left(z^{\prime}\right)\right\rangle=(1 / \gamma) L\left(z-z^{\prime}\right)$, where $L(z)=\int e^{i q \cdot z} l\left(k^{2}\right) g\left(k^{2}\right) d k$.

Since we need $\left\langle\varrho(z) \varrho\left(z^{\prime}\right)\right\rangle$ to be a tempered distribution we must make assumptions: We require $l\left(k^{2}\right) g\left(k^{2}\right)$ to be a polynomially bounded function.

Under this condition $\omega(z)=\varrho(z)+i \eta(z)$ is defined satisfying (5.11) and (5.12).

To finish we must construct the anticommuting fields $\psi$ and $\bar{\psi}$ satisfying (5.9), (5.10), (5.8), and (5.13). The last two conditions just say $\psi$ and $\bar{\psi}$ are independent of $\varphi$ and $\eta$.

We will use Osterwalder and Schrader's method for constructing Euclidean Fermi fields [14]. Let $b^{*}(z), b(z)$ and $d^{*}(z), d(z)$ be two independent sets of 
anticommuting creation and annihilation operators:

$$
\begin{aligned}
\left\{b(z), b\left(z^{\prime}\right)\right\} & =\left\{b^{*}(z), b^{*}\left(z^{\prime}\right)\right\}=0, \\
\left\{d(z), d\left(z^{\prime}\right)\right\} & =\left\{d^{*}(z), d^{*}\left(z^{\prime}\right)\right\}=0, \\
\left\{b(z), d\left(z^{\prime}\right)\right\}=\left\{b(z), d^{*}\left(z^{\prime}\right)\right\} & =\left\{b^{*}(z), d\left(z^{\prime}\right)\right\}=\left\{b^{*}(z), d^{*}\left(z^{\prime}\right)\right\}=0,
\end{aligned}
$$

such that

$$
\left\{b(z), b^{*}\left(z^{\prime}\right)\right\}=\left\{d(z), d^{*}\left(z^{\prime}\right)\right\}=-4 s^{\prime}\left(\left(z-z^{\prime}\right)^{2}\right) .
$$

Define

$$
\psi(z)=b^{*}(z)+d(z), \quad \bar{\psi}(z)=d^{*}(z)-b(z) .
$$

Then

$$
\left\{\psi(z), \psi\left(z^{\prime}\right)\right\}=\left\{\bar{\psi}(z), \bar{\psi}\left(z^{\prime}\right)\right\}=\left\{\psi(z), \bar{\psi}\left(z^{\prime}\right)\right\}=0 .
$$

Let $\mathscr{K}$ be the Fock Hilbert space for the $b^{*}(z), b(z), d^{*}(z), d(z)$, and let $\Omega$ be the vacuum. Let $\langle A\rangle=(\Omega, A \Omega)$ for an operator $A$ on $\mathscr{K}$.

Then

$$
\begin{aligned}
& \left\langle\psi(z) \psi\left(z^{\prime}\right)\right\rangle=\left\langle\bar{\psi}(z) \bar{\psi}\left(z^{\prime}\right)\right\rangle=0, \\
& \left\langle\psi(z) \bar{\psi}\left(z^{\prime}\right)\right\rangle=-4 s^{\prime}\left(\left(z-z^{\prime}\right)^{2}\right) .
\end{aligned}
$$

Having defined the fields $\varphi(z), \omega(z), \bar{\psi}(z), \psi(z)$ satisfying (5.7)-(5.13) we can define the superfield $\Phi(z, \theta, \bar{\theta})$ by (5.6). By construction we have (5.5). It turns out that (5.4) also holds.

\section{Supersymmetric Interactions}

We now restrict ourselves to the case when $\varphi(z)$ is the Euclidean free field in $D$ dimensions with covariance

$$
\left\langle\varphi(z) \varphi\left(z^{\prime}\right)\right\rangle=\gamma G_{2+\varepsilon, \chi}\left(z-z^{\prime}\right),
$$

where $\gamma, \varepsilon>0$ and $G_{2+\varepsilon, \chi}(z)$ is given by (2.2).

$G_{2+\varepsilon, \chi}(z)$ is not rotation invariant because of the $\chi$-cutoff, but it is invariant under rotations of the $y$ variables. We can thus define

$$
S_{\varepsilon}\left(x, y^{2}\right)=G_{2+\varepsilon, \chi}(x, y) .
$$

The discussion of the last section is still valid and we can construct a superfield $\Phi(z, \theta, \bar{\theta})$ with covariance

$$
s_{\varepsilon}\left(x-x^{\prime},\left(y-y^{\prime}\right)^{2}+\left(\bar{\theta}-\overline{\theta^{\prime}}\right)\left(\theta-\theta^{\prime}\right)\right),
$$

except that the expectation functions of the superfield are now only invariant with respect to supersymmetries in the $y, \theta, \bar{\theta}$ variables.

From now on supersymmetry will mean supersymmetries in the $y, \theta, \bar{\theta}$ variables. 
If we let $s_{\varepsilon}^{\prime}\left(x, y^{2}\right)=\left.\frac{\partial}{\partial r} s_{\varepsilon}(x, r)\right|_{r=y^{2}}$, it follows from Lemma 5.1 that

$$
-4 s_{\varepsilon}^{\prime}\left(x, y^{2}\right)=(1+\varepsilon)^{-1} G_{1+\varepsilon, \chi}(x, y) .
$$

Thus the superfield

$$
\Phi(z, \theta, \bar{\theta})=\varphi(z)+\bar{\psi}(z) \theta+\bar{\theta} \psi(z)+\theta \bar{\theta} \omega(z)
$$

is defined with $\varphi(z)$ given by $(6.1)$,

$$
\left\langle\psi(z) \bar{\psi}\left(z^{\prime}\right)\right\rangle=(1+\varepsilon)^{-1} G_{1+\varepsilon, \chi}\left(z-z^{\prime}\right),
$$

and

$$
\omega(z)=[\gamma(1+\varepsilon)]^{-1}\left(-\Delta+m^{2}\right) \varphi(z)+i \eta(z),
$$

where

$$
\left\langle\eta(z) \eta\left(z^{\prime}\right)\right\rangle=\gamma^{-1}(1+\varepsilon)^{-2} G_{\varepsilon, \chi}\left(z-z^{\prime}\right) .
$$

The reason for introducing the extra cutoff $\varepsilon$ is that $G_{1+\varepsilon, \chi}(0)$ is finite for $\varepsilon>0$ so $\psi(z), \bar{\psi}(z)$ are bounded operators and we can avoid technical difficulties when integrating over anticommuting variables.

The precise form of the cutoff comes from requiring $l(-\Delta)$ (defined in Sect. 5) to be equal to $-\Delta+m^{2}$ up to multiplication by a constant. This is needed so we can use the proof of the integrability of $\widetilde{\mathscr{H}}_{2, \chi}(\varphi ; f)$ of Sect. 3.

In Sect. 8 we will let $\varepsilon \rightarrow 0$ to obtain Theorem II.

Let us recall some facts about anticommuting fields. We have

$$
\|\psi(z)\| \leqq\left[2(1+\varepsilon)^{-1} G_{1+\varepsilon, \chi}(0)\right]^{1 / 2} \equiv \alpha<\infty,
$$

where $\alpha$ depends on $\varepsilon$ and $\chi ;\|\|$ is the operator norm in the Fock Hilbert space $\mathscr{K}$. Moreover, $z \rightarrow \psi(z)$ is a continuous function in the operator norm topology, we actually have

$$
\left\|\psi(z)-\psi\left(z^{\prime}\right)\right\| \leqq 2\left[(1+\varepsilon)^{-1}\left(G_{1+\varepsilon, \chi}(0)-G_{1+\varepsilon, \chi}\left(z-z^{\prime}\right)\right)\right]^{1 / 2} .
$$

Those results and estimates also hold for $\bar{\psi}(z)$. Thus, if $g \in L^{1}\left(\mathbb{R}^{D}, d z\right)$, $\int \bar{\psi}(z) \psi(z) g(z) d z$ is well defined as a bounded operator on $\mathscr{K}$, and

$$
\left\|\int \bar{\psi}(z) \psi(z) g(z) d z\right\| \leqq \alpha^{2}\|g\|_{1} .
$$

We can thus define $\exp \left\{-\int \bar{\psi}(z) \psi(z) g(z) d z\right\}$ as a bounded operator on $\mathscr{K}$, and

$$
\left\|\exp \left\{-\int \bar{\psi}(z) \psi(z) g(z) d z\right\}\right\| \leqq \exp \left\{\alpha^{2}\|g\|_{1}\right\} .
$$

We recall that ([13] for the finite dimensional case; the extension to the present case is straightforward since all operators are bounded)

$$
\left\langle\exp \left\{-\int \bar{\psi}(z) \psi(z) g(z) d z\right\}\right\rangle=\operatorname{det}\left(I+(1+\varepsilon)^{-1} G_{(1+\varepsilon / 2), \chi^{1 / 2}} g G_{(1+\varepsilon / 2), \chi^{1 / 2}}\right),
$$

where as before we denote by $G_{s, \chi}$ the operator with kernel $G_{s, \chi}\left(z-z^{\prime}\right)$. Notice that

$$
T_{r}\left[\mid G_{(1+\varepsilon / 2), \chi^{1 / 2}} g G_{\left.(1+\varepsilon / 2), \chi^{1 / 2} \mid\right] \leqq G_{1+\varepsilon, \chi}(0)\|g\|_{1}<\infty}\right.
$$

so the determinant is well defined. 
We are now ready to define polynomials in the superfield. Let $P$ be a polynomial in one variable. Then

$$
\begin{aligned}
P(\Phi(z, \theta, \bar{\theta}))= & P(\varphi(z))+P^{\prime}(\varphi(z)) \bar{\psi}(z) \theta+P^{\prime}(\varphi(z)) \bar{\theta} \psi(z) \\
& +\left[P^{\prime}(\varphi(z)) \omega(z)+P^{\prime \prime}(\varphi(z)) \bar{\psi}(z) \psi(z)\right] \theta \bar{\theta} .
\end{aligned}
$$

All the terms are well defined as they stand except for the term involving $\omega(z)$ which must be defined in the distributional sense.

So let $F(z, \theta, \bar{\theta})=F_{0}(z)+F_{1}(z) \theta+F_{2}(z) \bar{\theta}+F_{3}(z) \bar{\theta} \theta$, and

$$
\begin{aligned}
P(\Phi, F)= & \int P(\Phi(z, \theta, \bar{\theta})) F(z, \theta, \bar{\theta}) d \bar{\theta} d \theta d z \\
= & \int P^{\prime}(\varphi(z)) \omega(z) F_{0}(z) d z+\int P^{\prime \prime}(\varphi(z)) \bar{\psi}(z) \psi(z) F_{0}(z) d z \\
& -\int P(\varphi(z)) F_{3}(z) d z+\int P^{\prime}(\varphi(z)) \bar{\psi}(z) F_{2}(z) d z-\int P^{\prime}(\varphi(z)) \psi(z) F_{1}(z) d z .
\end{aligned}
$$

We will use $L^{p}$ norms in the commutative variables and operator norms in the anticommuting variables. Our independent commutative variables are $\varphi$ and $\eta$, let $(Q, d \lambda(\varphi, \eta))$ be the underlying probability space. Let $\mathscr{B}(\mathscr{K})$ the Banach space of bounded operator on $\mathscr{K}$. We will define $P(\Phi, F)$ as an element of $L^{p}(Q, \mathscr{B}(\mathscr{K}) ; d \lambda)$ for $1 \leqq p<\infty$. From (6.8), (6.1), (6.3), (6.4), (6.5), (6.6), it follows that it suffices to require $F_{0} \in L^{1} \cap L^{2}, F_{1}, F_{2}, F_{3} \in L^{1}$, and that

$$
\left[\int\|P(\Phi, F)\|^{p} d \lambda(\varphi, \eta)\right]^{1 / p} \leqq C\left[\left\|F_{0}\right\|_{1}+\left\|F_{0}\right\|_{2}+\left\|F_{1}\right\|_{1}+\left\|F_{2}\right\|_{1}+\left\|F_{3}\right\|_{1}\right]
$$

for some finite constant $C$ that depends only on $P, p$, and $\varepsilon\left(\gamma, m^{2}, \chi\right.$, etc. being fixed). If $F$ is supersymmetric, it follows from Proposition 4.1 that $F(z, \theta, \bar{\theta})$ $=\tilde{f}\left(x, y^{2}+(4 / \gamma) \bar{\theta} \theta\right)$, where $\quad f(x, y)=\tilde{f}\left(x, y^{2}\right)=F_{0}(x, y), \quad$ and $f^{\prime}(x, y)$ $=\left.\frac{\partial}{\partial t} \tilde{f}(x, t)\right|_{t=y^{2}}$. In this case we will write $P(\Phi, f)$ for $P(\Phi, F)$, and

$$
P(\Phi, f)=\int P^{\prime}(\varphi(z)) \omega(z) f(z) d z+\int P^{\prime \prime}(\varphi(z)) \bar{\psi}(z) \psi(z) f(z) d z-(4 / \gamma) \int P(\varphi(z)) f^{\prime}(z) d z .
$$

Thus

$$
\begin{aligned}
P(\Phi, f)= & {[\gamma(1+\varepsilon)]^{-1} \int\left(-\Delta+m^{2}\right) \varphi(z) P^{\prime}(\varphi(z)) f(z) d z } \\
& +i \int P^{\prime}(\varphi(z)) \eta(z) f(z) d z+\int P^{\prime \prime}(\varphi(z)) \bar{\psi}(z) \psi(z) f(z) d z \\
& -(4 / \gamma) \int P(\varphi(z)) f^{\prime}(z) d z .
\end{aligned}
$$

Let now $V$ be a bounded below polynomial of degree $\delta$ and $f$ a $m^{2} \delta$ appropriate space cutoff as in Theorem I. Let us consider the function $e^{-V(\Phi, f)}$. Then

$$
\begin{aligned}
\left\|e^{-V(\Phi, f)}\right\| \leqq & \exp \left\{-[\gamma(1+\varepsilon)]^{-1} \int\left(-\Delta+m^{2}\right) \varphi(z) V^{\prime}(\varphi(z)) f(z) d z\right. \\
& \left.+(4 / \gamma) \int P(\varphi(z)) f^{\prime}(z) d z+\alpha^{2} \int\left|P^{\prime \prime}(\varphi(z))\right| f(z) d z\right\} \equiv \mathscr{V}(\varphi ; f) .
\end{aligned}
$$

By the methods of Sect. 3, $\mathscr{V}(\varphi, f)$ is in all $L^{p}$ for $1 \leqq p<\infty$.

For a function $\Gamma(\Phi)$ of the superfield let $\langle\Gamma(\Phi)\rangle=\int(\Omega, \Gamma(\Phi) \Omega) d \lambda(\varphi, \eta)$. We will be concerned with expectations of the form $\left\langle\Gamma(\Phi) e^{-V(\Phi, f)}\right\rangle$. Notice that

$$
\left|\left\langle\Gamma(\Phi) e^{-V(\Phi, f)}\right\rangle\right| \leqq\left\langle\|\Gamma(\Phi)\|\left\|\mathrm{e}^{-V(\Phi, f)}\right\|\right\rangle \leqq\|\| \Gamma(\phi)\|\|_{p}\|\mathscr{V}(\varphi, f)\|_{p^{\prime}}
$$

for $\frac{1}{p}+\frac{1}{p^{\prime}}=1$. 


\section{The Dimensional Reduction for Supersymmetric Theories}

We keep the framework of Sect. 6. In particular, supersymmetries act only on the $y, \theta, \bar{\theta}$ variables and leave the $x$ variables fixed. Thus any function of $\varphi(x, 0)$ is supersymmetric.

Theorem III. Let $\Phi(z, \theta, \bar{\theta})$ be the superfield defined in Sect. 6 and let $V$ be $a$ bounded below polynomial of degree $\delta$ and $f a^{2} \delta$-appropriate space cutoff as in Theorem $I$. Let $\Gamma(\Phi)$ be a function of the superfield which is left invariant by all supersymmetries in $y, \theta, \bar{\theta}$, such that $\|\Gamma(\Phi)\| \in L^{p_{0}}$ for some $p_{0}>1$. Then

$$
\begin{aligned}
& \left\langle\Gamma(\Phi) \exp \left\{-\int V(\Phi(z, \theta, \bar{\theta})) \tilde{f}\left(x, y^{2}+(4 / \gamma) \bar{\theta} \theta\right) d \bar{\theta} d \theta d z\right\}\right\rangle \\
& \quad=\left\langle\Gamma(\Phi) \exp \left\{-(4 \pi / \gamma) \int V(\varphi(x, 0)) \tilde{f}(x, 0) d x\right\}\right\rangle . \quad \square
\end{aligned}
$$

Proof. Let

$$
\begin{gathered}
V(\Phi, f)=\int V(\Phi(z, \theta, \bar{\theta})) \tilde{f}\left(x, y^{2}+(4 / \gamma) \bar{\theta} \theta\right) d \bar{\theta} d \theta d z, \\
V(\varphi, f)=\int V(\varphi(x, 0)) \tilde{f}(x, 0) d x .
\end{gathered}
$$

For $0 \leqq s \leqq 1$ and any function $G(\Phi)$ let

$$
\{G(\Phi)\}_{s}=\langle G(\Phi) \Gamma(\Phi) \exp \{-(1-s) V(\Phi, f)-s(4 \pi / \gamma) V(\varphi, f)\}\rangle .
$$

This is well defined if $\|G(\Phi)\| \in L^{q_{0}}$ for some $1<q_{0}$ such that $\frac{1}{p_{0}}+\frac{1}{q_{0}}<1$.

We define $g(s)=\{1\}_{s}$. To prove the theorem we must show that $g(0)=g(1)$. Since $\|\Gamma(\Phi)\| \in L^{p_{0}}$ with $p_{0}>1$ and $\|V(\Phi, f)\|, V(\varphi, f)$, and $\| \exp \{-(1-s) V(\Phi, f)$ $-s(4 \pi / \gamma) V(\varphi, f)\} \|$ are in all $L^{p}$ for $1 \leqq p<\infty$, we can differentiate under the expectation and get

$$
g^{\prime}(s)=\{V(\Phi, f)\}_{s}-(4 / \gamma)\{V(\varphi, f)\}_{s} .
$$

We will use Lemma 4.5 to conclude that $g^{\prime}(s)=0$ for all $0 \leqq s \leqq 1$ and hence that $g(0)=g(1)$.

Let us consider the distribution $T(z, \theta, \bar{\theta})$ given by $T(F)=\{V(\Phi, F)\}_{s}$, where $F(z, \theta, \bar{\theta})$ has $F_{0} \in L^{1} \cap L^{2}$ and $F_{1}, F_{2}, F_{3} \in L^{1} . T(F)$ is well defined and a bound like (6.10) holds.

Moreover, since $V(\Phi, f)$ and $\Gamma(\Phi)$ are supersymmetric in $y, \theta, \bar{\theta}$, it follows from the supersymmetry of the expectations of the superfield $\Phi$ that $T$ is supersymmetric in $y, \theta, \bar{\theta}$. Thus, we have from Proposition 4.4 that $T(F)=T_{3}\left(F_{0}\right)-T_{0}\left(F_{3}\right)$, and it follows from the (6.10)-like estimate that $|T(F)| \leqq C\left(\left\|F_{0}\right\|_{1}+\left\|F_{0}\right\|_{2}+\left\|F_{3}\right\|_{1}\right)$, for some finite constant $C$.

Moreover, from (6.9) we have that

$$
T_{0}\left(F_{3}\right)=\left\{\int V(\varphi(z)) F_{3}(z) d z\right\}_{s}=\int\{V(\varphi(z))\}_{s} F_{3}(z) d z,
$$

since $V(\varphi(z))$ is a bounded continuous function of $z \in \mathbb{R}^{D}$ with values in $L^{p}$ for $1 \leqq p<\infty$. Thus $T_{0}$ is given by a bounded continuous function $T_{0}(z)=\{V(\varphi(z))\}_{s}$.

We can now apply Lemma 4.5 to $T$ and to the function $\tilde{f}\left(x, y^{2}+(4 / \gamma) \bar{\theta} \theta\right)$ to conclude that

$$
\{V(\Phi, f)\}_{s}=(4 \pi / \gamma) \int\{V(\varphi(x, 0))\}_{s} f(x, 0) d x=(4 \pi / \gamma)\{V(\varphi, f)\}_{s} .
$$

Thus it follows from (7.2) that $g^{\prime}(s)=0$. 


\section{Proof of Theorem II}

Let $\varphi(z)$ be the Euclidean free field in $D$ dimensions with covariance given by (6.1); we will denote by $d \mu_{\chi, \varepsilon}(\varphi)$ its probability distribution.

For such $\varphi(z)$ we constructed the corresponding superfield $\Phi(z, \theta, \bar{\theta})$ and proved Theorem III. If we now take $\Gamma(\Phi)$ to be some function of $\{\varphi(x, 0)$; $\left.x \in \mathbb{R}^{D-2}\right\}$ [e.g., $\left.\varphi\left(x_{1}, 0\right) \ldots \varphi\left(x_{n}, 0\right)\right]$ which we will write $\Gamma(\varphi(\cdot, 0))$ we can rewrite both sides of (7.1) as functional integrals over $\varphi$.

We have

$$
\begin{aligned}
&\left\langle\Gamma(\varphi(\cdot, 0)) e^{-V(\Phi, f)}\right\rangle \\
&=\left\langle\Gamma \left(\varphi ( \cdot , 0 ) \operatorname { e x p } \left\{-[\gamma(1+\varepsilon)]^{-1} \int\left(-\Delta+m^{2}\right) \varphi(z) V^{\prime}(\varphi(z)) f(z) d z\right.\right.\right. \\
&\left.\left.-i \int V^{\prime}(\varphi(z)) \eta(z) f(z) d z-\int V^{\prime \prime}(\varphi(z)) \bar{\psi}(z) \psi(z) f(z) d z+(4 / \gamma) \int V(\varphi(z)) f^{\prime}(z) d z\right\}\right\rangle \\
&=\left\langle\Gamma \left(\varphi ( \cdot , 0 ) \operatorname { e x p } \left\{-[\gamma(1+\varepsilon)]^{-1} \int\left(-\Delta+m^{2}\right) \varphi(z) V^{\prime}(\varphi(z)) f(z) d z\right.\right.\right. \\
&\left.+(4 / \gamma) \int V(\varphi(z)) f^{\prime}(z) d z\right\}\left\langle\exp \left\{-i \int V^{\prime}(\varphi(z)) \eta(z) f(z) d z\right\}\right\rangle_{\eta} \\
&\left.\cdot\left(\Omega, \exp \left\{-\int V^{\prime \prime}(\varphi(z)) \bar{\psi}(z) \psi(z) f(z) d z\right\} \Omega\right)\right\rangle_{\varphi} .
\end{aligned}
$$

But since $V^{\prime}(\varphi(z)) f(z) \in L^{2}$ for $\mu_{\chi, \varepsilon}(\varphi)$-a.e. $\varphi$, we get from (6.4) that

$$
\begin{aligned}
& \left\langle\exp \left\{-i \int V^{\prime}(\varphi(z)) \eta(z) f(z) d z\right\}\right\rangle_{\eta} \\
& \quad=\exp \left\{-(2 \gamma)^{-1}(1+\varepsilon)^{-2} \iint V^{\prime}(\varphi(z)) V^{\prime}\left(\varphi\left(z^{\prime}\right)\right) f(z) f\left(z^{\prime}\right) G_{\varepsilon, \chi}\left(z-z^{\prime}\right) d z d z^{\prime}\right\} .
\end{aligned}
$$

From (6.8), we get

$$
\begin{aligned}
& \left(\Omega, \exp \left\{-\int V^{\prime \prime}(\varphi(z)) \bar{\psi}(z) \psi(z) f(z) d z\right\} \Omega\right) \\
& \quad=\operatorname{det}\left(I+(1+\varepsilon)^{-1} G_{(1+\varepsilon / 2), \chi^{1 / 2}} V^{\prime \prime}(\varphi) f G_{(1+\varepsilon / 2), \chi^{1{ }^{\prime} 2}}\right)
\end{aligned}
$$

for $\mu_{\chi, \varepsilon}(\varphi)-$ a.e. $\varphi$. Thus

$$
\left\langle\Gamma(\varphi(\cdot, 0)) e^{-V(\Phi, f)}\right\rangle=\int \Gamma(\varphi) \tilde{\mathscr{H}}_{\chi, \varepsilon}(\varphi ; f) d \mu_{\chi, \varepsilon}(\varphi),
$$

where

$$
\begin{aligned}
& \tilde{\mathscr{H}}_{\chi, \varepsilon}(\varphi ; f)=\exp \left\{(4 / \gamma) \int V(\varphi(z)) f^{\prime}(z) d z\right\} \mathscr{H}_{\chi, \varepsilon}(\varphi ; f), \\
\mathscr{H}_{\chi, \varepsilon}(\varphi ; f)= & \exp \left\{-(2 \gamma)^{-1}(1+\varepsilon)^{-2} \iint V^{\prime}(\varphi(z)) V^{\prime}\left(\varphi\left(z^{\prime}\right)\right) f(z) f\left(z^{\prime}\right) G_{\varepsilon, \chi}\left(z-z^{\prime}\right) d z d z^{\prime}\right. \\
& \left.-[\gamma(1+\varepsilon)]^{-1} \int\left(-\Delta+m^{2}\right) \varphi(z) V^{\prime}(\varphi(z)) f(z) d z\right\} \\
& \cdot \operatorname{det}\left(I+(1+\varepsilon)^{-1} G_{(1+\varepsilon / 2), \chi^{1 / 2}} V^{\prime \prime}(\varphi) f G_{(1+\varepsilon / 2), \chi^{1 / 2}}\right) .
\end{aligned}
$$

Now let us rewrite the right-hand-side of (7.1). From (6.1), (2.2), (2.4), we get by a simple integration that

$$
\left\langle\varphi(x, 0) \varphi\left(x^{\prime}, 0\right)\right\rangle=(\gamma / 4 \pi) H_{1+\varepsilon, \chi}\left(x-x^{\prime}\right) .
$$

Thus let $\phi(x)=\varphi(x, 0)$, and let $d v_{\chi, \varepsilon}(\phi)$ be its probability distribution. Then

$$
\left\langle\Gamma(\varphi(\cdot, 0)) e^{-(4 \pi / \gamma) V(\varphi, f)}\right\rangle=\int \Gamma(\phi) \exp \left\{-(4 \pi / \gamma) \int V(\phi(x)) f(x, 0) d x\right\} d v_{\chi, \varepsilon}(\phi) .
$$

Thus Theorem III says that

$$
\int \Gamma(\varphi(\cdot, 0)) \mathscr{H}_{\chi, \varepsilon}(\varphi ; f) d \mu_{\chi, \varepsilon}(\varphi)=\int \Gamma(\phi) \exp \left\{-(4 \pi / \gamma) \int V(\phi(x)) f(x, 0) d x\right\} d v_{\chi, \varepsilon}(\phi) .
$$

To get Theorem II we need only to let $\varepsilon \rightarrow 0$. 
To do this let us fix the Gaussian generalized process $\left\{\varphi(z) ; z \in \mathbb{R}^{D}\right\}$ with covariance $\left\langle\varphi(z) \varphi\left(z^{\prime}\right)\right\rangle=\gamma G_{2, \chi}\left(z-z^{\prime}\right)$. Its probability distribution is $d \mu_{\chi}(\varphi)$. Let $\varphi_{\varepsilon}(z)=\left[\left(-\Delta+m^{2}\right)^{-\varepsilon / 2} \varphi\right](z)$. Then

$$
\int \varphi_{\varepsilon}(z) \varphi_{\varepsilon}\left(z^{\prime}\right) d \mu_{\chi}(\varphi)=\gamma G_{2+\varepsilon, \chi}\left(z-z^{\prime}\right) ;
$$

i.e., the probability distribution of $\varphi_{\varepsilon}$ is $d \mu_{\chi, \varepsilon}$. Thus the left-hand-side of (8.1) is equal to

$$
\int \Gamma\left(\varphi_{\varepsilon}(\cdot, 0)\right) \tilde{\mathscr{H}}_{\chi, \varepsilon}\left(\varphi_{\varepsilon} ; f\right) d \mu_{\chi}(\varphi) .
$$

Similarly, let $\left\{\phi(\mathrm{x}), x \in \mathbb{R}^{D-2}\right\}$ be the Gaussian generalized process with covariance $\left\langle\phi(x) \phi\left(x^{\prime}\right)\right\rangle=(\gamma / 4 \pi) H_{1, \chi}\left(x-x^{\prime}\right)$. Its probability distribution is $d v_{\chi}(\phi)$. Define $\phi_{\varepsilon}(x)=\left[\left(-\Delta+m^{2}\right)^{-\varepsilon / 2} \phi\right](x)$. Then $\int \phi_{\varepsilon}(x) \phi_{\varepsilon}\left(x^{\prime}\right) d v_{x}(\phi)=(4 \pi / \gamma) H_{1+\varepsilon, \chi}(\phi)$; i.e., the probability distribution of $\phi_{\varepsilon}$ is $d v_{\chi, \varepsilon}$. The right-hand-side of (8.1) is thus equal to

$$
\int \Gamma\left(\phi_{\varepsilon}\right) \exp \left[-(4 \pi / \gamma) \int V\left(\phi_{\varepsilon}(x)\right) f(x, 0) d x\right\} d v_{\chi}(\phi) .
$$

Thus (8.1) can be rewritten as

$$
\int \Gamma\left(\varphi_{\varepsilon}(\cdot, 0)\right) \tilde{\mathscr{H}}_{\chi, \varepsilon}\left(\varphi_{\varepsilon} ; f\right) d \mu_{\chi}(\varphi)=\int \Gamma\left(\phi_{\varepsilon}\right) \exp \left\{-(4 \pi / \gamma) \int V\left(\phi_{\varepsilon}(x)\right) f(x, 0) d x\right\} d v_{\chi}(\phi) .
$$

Now, let $\Gamma\left(\varphi_{\varepsilon}(\cdot, 0)\right)$ be such that $\Gamma\left(\varphi_{\varepsilon}(\cdot, 0)\right) \rightarrow \Gamma(\varphi(\cdot, 0))$ in some $L^{p}\left(d \mu_{\chi}(\varphi)\right)$ with $1<p$, e.g., $\Gamma\left(\varphi_{\varepsilon}(\cdot, 0)\right)=\varphi_{\varepsilon}\left(x_{1}, 0\right) \ldots \varphi_{\varepsilon}\left(x_{n}, 0\right)$. By standard methods (e.g., $\left.[11,12]\right)$

$$
\exp \left\{-(4 \pi / \gamma) \int V\left(\phi_{\varepsilon}(x)\right) f(x, 0) d x\right\} \rightarrow \exp \left\{(-4 \pi / \gamma) \int V(\phi(x)) f(x, 0) d x\right\}
$$

in $L^{p}\left(d v_{\chi}(\phi)\right)$ as $\varepsilon \rightarrow 0$ for all $1 \leqq p<\infty$.

We can break down $\tilde{\mathscr{H}}_{\chi, \varepsilon}\left(\varphi_{\varepsilon} ; f\right)$ as

$$
\tilde{\mathscr{H}}_{\chi, \varepsilon}\left(\varphi_{\varepsilon} ; f\right)=\mathscr{H}_{\chi, \varepsilon, 1}(\varphi ; f) \tilde{\mathscr{H}}_{\chi, \varepsilon, 2}(\varphi ; f) \tilde{\mathscr{H}}_{\chi, \varepsilon, 3}(\varphi ; f) \mathscr{H}_{\chi, \varepsilon, 4}(\varphi ; f)
$$

in the same way as in Sect. 3. Again standard methods as used in Sect. 3 (e.g., $[11,12])$ give convergence in $L^{p}\left(d \mu_{\chi}(\varphi)\right)$ as $\varepsilon \rightarrow 0,1 \leqq p<\infty$, of each factor in $(8.3)$ to the similar factor without the $\varepsilon$. Thus we can conclude that $\widetilde{\mathscr{H}}_{\chi, \varepsilon}\left(\varphi_{\varepsilon} ; f\right) \rightarrow \tilde{\mathscr{H}}_{\chi}(\varphi ; f)$ in $L^{p}\left(d \mu_{\chi}(\varphi)\right)$ as $\varepsilon \rightarrow 0$, for all $1 \leqq p<\infty$.

We can now take the limit as $\varepsilon \rightarrow 0$ in (8.2) to obtain

$$
\begin{aligned}
& \int \varphi\left(x_{1}, 0\right) \ldots \varphi\left(x_{n}, 0\right) \tilde{\mathscr{H}}_{\chi}(\varphi ; f) d \mu_{\chi}(\varphi) \\
& \quad=\int \phi\left(x_{1}\right) \ldots \phi\left(x_{n}\right) \exp \left\{-(4 \pi / \gamma) \int V(\phi(x)) f(x, 0) d x\right\} d v_{\chi}(\phi) .
\end{aligned}
$$

This completes the proof of Theorem II.

\section{References}

1. Imry, Y., Ma, S.K.: Random field instability of the ordered state of continuous symmetry. Phys. Rev. Lett. 35, 1399-1401 (1976)

2. Grinstein, G.: Ferromagnetic phase transition in random fields: the breakdown of scaling laws. Phys. Rev. Lett. 37, 944-947 (1976)

3. Aharony, A., Imry, Y., Ma, S.K.: Lowering of dimensionality in phase transitions with random fields. Phys. Rev. Lett. 37, 1364-1367 (1976) 
4. Young, A.P.: On the lowering of dimensionality in phase transitions with random fields. J. Phys. C 10, L257-L262 (1977)

5. Parisi, G., Sourlas, N.: Random magnetic fields, supersymmetry, and negative dimensions. Phys. Rev. Lett. 43, 744-745 (1979)

6. Niemi, A.: Disorder solitons. Phys. Rev. Lett. 49, 1808-1811 (1982)

7. McClain, B., Niemi, A., Taylor, C., Wijewardhana, L.C.R.: Superspace, dimensional reduction, and stochastic quantization. Nucl. Phys. B 217, 430-460 (1983)

8. Klein, A., Perez, J.F.: Supersymmetry and dimensional reduction: a non-perturbative proof. Phys. Lett. 125 B, 473-475 (1983)

9. Cardy, J.L.: Nonperturbative effects in a scalar supersymmetric theory. Phys. Lett. 125 B, 470-472 (1983)

10. Parisi, G., Sourlas, N.: Supersymmetric field theories and stochastic differential equations. Nucl. Phys. B 206, 321-332 (1982)

11. Glimm, J., Jaffe, A.: Quantum physics. Berlin, Heidelberg, New York: Springer 1981

12. Simon, B.: The $P(\phi)_{2}$ Euclidean (quantum) field theory. Princeton, NJ: Princeton University Press 1974

13. Berezin, F.A.: The method of second quantization. New York: Academic Press 1966

14. Osterwalder, K., Schrader, R.: Euclidean Fermi fields and a Feynman-Kac formula for BosonFermion models. Helv. Phys. Acta 46, 277-302 (1973)

Communicated by A. Jaffe

Received November 21, 1983; in revised form March 26, 1984 